\title{
An overstability analysis of vertically vibrated suspension of active swimmers subjected to thermal stratification
}

\author{
Virendra Kumar $^{1} \cdot$ K. Srikanth ${ }^{1}$ \\ Received: 9 October 2020 / Accepted: 31 March 2021 \\ Published online: 10 May 2021 \\ (c) The Author(s) 2021 \\ OPEN
}

\begin{abstract}
The present article focuses on the analytical approach to discuss the thermo-vibrational convection in a suspension of the active (gyrotactic) swimmers. The onset of instability criterion is investigated for the stationary and oscillatory modes of convection in a shallow fluid layer with no-slip and rigid-free walls. The eigenvalue problem is tackled by Galerkin scheme to get the desired stability diagram and the correlation between the critical Rayleigh numbers. The overstability in suspension is possible when the unstable density gradient of the gyrotactic particles is opposed by the density variation due to thermo-vibrational influence. The suspension is destabilized due to gyrotactic up-swimming while the increase in Péclet number stabilizes the system. The stabilizing influence of vertical vibration is considerably affected due to thermal gradient which destabilizes the suspension. An interesting result of this study is the influence of thermo-vibrational parameter which is associated with applied thermal and vibrational properties. We reported that the destabilizing nature of thermo-vibrational parameter becomes thermally or vibrationally governed when the suspension is heated or cooled from below. When compared to the rigid-rigid boundaries, the displayed profiles for rigid-free walls yielded less stableness in the suspension.
\end{abstract}

Keywords Overstability · Thermo-vibrational bioconvection · Vertical vibration · Gyrotactic · Rigid-free boundaries

\section{Introduction}

Bioconvection in a suspension of swimming active particles is the emerging phenomena that have significantly attracted the scientific community to put a considerable interest in the development of mesoscopic/microscopic pattern formations [1-4]. Bioconvection illustrates a mechanism for inducing the mass transfer mixing in the fluid micro-volumes and is potentially applicable in several pharmaceutical and bioengineering technologies $[5,6]$. A numerical computation for three dimensional gyrotactic plumes in a rectangular chamber with free-free sidewalls with their structural formations was scripted by Ghorai and Singh [7]. A classical linear stability theory is available on spheroidal orientations for uniform gyrotactic bioconvection [8]. For a uniformly sheared and down-flowing suspension Hwang and Pedley analyzed the gyrotactic bioconvection in a two dimensional vertical flow $[9,10]$. Bio-thermal convection, unlike the conventional bioconvection, is induced by the self-propulsion of swimming micro-organisms and the imposed thermal gradient across the fluid medium [11]. Convective modeling of vertically oscillated systems of various active swimmers is an area of considerable interest which has motivated the researchers due to its enormous application in vibration production processes in specific oil industries [12, 13]. Zen'kovskaya and Simonenko studied the thermal vibrational convection and derived the time averaged

Virendra Kumar, virendrakumar@cutn.ac.in; K. Srikanth, srikanthehow@gmail.com | Department of Mathematics, School of Mathematics and Computer Sciences, Central University of Tamil Nadu, Thiruvarur 610005, India.

SN Applied Sciences $\quad$ (2021) 3:612 | https://doi.org/10.1007/s42452-021-04545-0 
equations for the system [14]. A classical theory of the dynamically stabilized viscous fluid-gas interface for a vertically oscillated fluid was presented by Wolf [15]. It was established that viscous properties of the interface exerts the stabilizing action for the short wave lengths. Lyubimov reported the impact of vibration on the convection excitation in the homogeneous fluid and discovered the stabilizing impact of vibration to the system [16]. The organized description for thermo-vibrational modeling and their stability results for Newtonian fluid can be found in the classical monograph of Gershuni and Lyubimov [17]. Zen'kovskaya and Novosiadliy reported the high-frequency vibrational analyses for the case of immiscible two layer suspension [18]. Recently, an influence of time periodic alternating acceleration on the dynamics of Rayleigh-Taylor convection is carried out by Boffetta and co-workers where they utilized the numerically simulated Boussinesq equations [19].

The modeling and solution of the linear stability problem of a vertically vibrated system of negatively geotactic swimmers in a dilute shallow medium is investigated by Kuznetsov [20]. Nield and Kuznetsov represented the extensive report on the oscillatory instability of bio-thermally stratified binary fluid with cooling surfaces [21]. Sharma and Kumar revisited and completed the analysis by discussing the problem of oscillatory convection in a porous saturated gyrotactic particles comprised with negative thermal gradient [22]. An overstability analysis of bio-thermal convection combining the nanoparticles and the gyrotactic swimmers is studied by Kuznetsov [23]. Furthermore, Sharma and Kumar expended the study by reporting the analytical computations for a vertically vibrated aqueous and porous suspension of the gyrotactic swimmers in the absence of the thermal effect [24, 25]. Becker and co-workers presented the experimental results to demonstrate the collective swimming of the bodies due to their flow mediated reaction [26]. A comprehensive review for the self propelled interactions of the active particles in a crowded and complex environment was reported by Bechinger and co-workers [27]. Uddin and co-workers discussed the nonlinear computations for the nanofluidic bioconvection affected by the multiple slips and Stefan blowing saturated by porous media [28]. Lovecchio et al. [29] studied the thermally stratified suspension of gyrotactic particles in a free surface turbulent channel and reported the various stability regimes numerically [29]. A nanofluidic bioconvection comprising of the variable viscosity and thermal conductivity within a rotating framework was investigated by Shuo and co-workers [30]. Moli Zhao and co-workers restudied the linear stability theory of thermo-bioconvection comprising the gyrotactic swimmers by utilizing the probabilistic continuum approach [31]. A numerically simulated gyrotactic reorientation affected by the wind-induced shear in free-surface turbulence was reported Mashayekhpour and co-workers [32]. Marchioli et al. [33] revisited and discussed the dynamics of gyrotactic swimmers in open channel turbulence using the Eulerian-Lagrangian simulations [33]. Nayak and co-workers performed the computations for the three-dimensional slip profiles of gyrotactic swimmers in the existence of chemically reacted nanofluid [34]. A detailed review addressing the successive developments and advances of the bioconvection theory is available in the literature [35].

From the above review of literature it is observed that there are no reports that describe the overstability results for the bio-thermal suspension of gyrotactic swimmers affected by the vertical vibration. Unstable density stratification and the applied temperature gradient across the vertically vibrated layer causes an interesting research problem making the suspension closer to the real flow problems. In this paper, a deterministic continuum approach for a binary gyrotactic bioconvection; located between two infinite horizontal surfaces, is extended to include the effect of thermal gradient and vertical vibration. This work is an augmentation to one of the author's previous reported study in the absence of thermal stratification, oscillatory convection and the rigid-free boundaries [24]. The adopted model consists of a shallow fluid layer and the physical mechanisms such as Boussinesq approximation are considered with no-slip and rigid-free boundaries. A detailed linear stability analysis describes a significant physical and mathematical insight to this thermo-vibrational convection. The intercession of the competing effects such as cooling from below, density distribution and the imposed vertical oscillations leads to the possibility of oscillatory convection. The dependency of bioconvection and wavelength profiles on the existing parameters provides the further discussion at the onset of instability problem.

\section{Formulation of the problem and analysis}

We assume an incompressible, electrically insulated, aqueous suspension of gyrotactic swimmers, restricted between infinite horizontal layers, $z=0$ and $z=d$. A high frequency low amplitude vibrational force acting vertically to the boundaries such that it doesn't affect the swimming speed and propulsion of the gyrotactic swimmers is considered. At the lower surface $(z=0)$ the temperature $T$ is kept at $T_{0}+\Delta T$ while at the upper surface $(z=d)$ it is maintained at $T_{0}$, which are assumed to be uniform. The considered intensity of thermal gradient is sufficiently weak so that the self-propelling nature of the swimmers remains unaltered. Cartesian system is adopted with 
assuming that layer is infinite in horizontal directions. The presented model is the deterministic continuum model developed for the gyrotactic swimmers [2]. The averaged equations for vibrational bioconvection in the high-frequency estimation were discussed and formulated [17, 24, 25]. In the present model, the conservation of momentum is supported by additional terms like vibrational and buoyancy forces resulted from the impacts of the vertical vibrations and thermal gradient. Under these approximations, we developed differential forms of physical laws of conservation of mass, momentum, number density concentration of the swimmers and the energy, respectively, as:

$\nabla \cdot \mathbf{u}=0$ characteristic times scales i.e. $\tau_{\text {vibration }}<<\operatorname{Min}\left(\tau_{\text {viscous }}, \tau_{\text {buoyancy, }} \tau_{\text {convective }}\right)$ [24]. The amplitude of vibration is sufficiently low which ultimately makes the quickly varying velocity components (in the inertial terms) negligibly small i.e., $\hat{b} \ll d / \theta \Delta \bar{T} \beta\left(\bar{n}_{1}-\bar{n}_{2}\right)\left(\Delta \rho / \rho_{0}\right)$ [37]. The quantity $\left(\bar{n}_{1}-\bar{n}_{2}\right)$ refers to the concentration difference of the active swimmers across the surface thickness and $\Delta \bar{T}$ is the temperature difference at the boundaries [17]. Under these conditions one set of equation describes the pulsation field while other one stands for the mean flow of the suspension. The time averaged equations for the mean flow is achieved by performing the order magnitude technique which enables the transformation of the rapid field variables to mean variables $[36,37]$. The

$\rho_{0}[\partial \mathbf{u} / \partial t+(\mathbf{u} \cdot \nabla) \mathbf{u}]=-\nabla p+\mu \nabla^{2} \mathbf{u}+\left[n \theta \Delta \rho-\beta\left(T-T_{0}\right) \rho_{0}\right] \mathbf{g}+\left[n \theta \Delta \rho+\beta\left(T-T_{0}\right) \rho_{0}\right]\left(\hat{\mathrm{b}} \omega^{2} \cos \omega t\right) \mathbf{k}$

$\partial n / \partial t=-\operatorname{div}\left[n \mathbf{u}+n q_{c} \hat{\mathbf{p}}-D_{c} \nabla n\right]$

$c_{p} \rho_{0}[\partial T / \partial t+(\mathbf{u} \cdot \nabla) T]=\kappa \nabla^{2} T$ constructed time averaged system (governing the flow) for the mean components are as follows:

$\nabla \cdot \overline{\mathbf{u}}=0$

$\rho_{0}[\partial \overline{\mathbf{u}} / \partial t+(\overline{\mathbf{u}} \cdot \nabla) \overline{\mathbf{u}}]=-\nabla \bar{p}+\mu \nabla^{2} \overline{\mathbf{u}}+\mathbf{g}\left[\bar{n} \theta \Delta \rho-\rho_{0} \beta \bar{T}\right]+\left(\rho_{0} / 2\right)\left[\left(\Delta \rho / \rho_{0}\right) \hat{\mathrm{b}} \theta \omega\right]^{2}\left(\overline{\mathbf{v}}_{1} \cdot \nabla\right)\left(\bar{n} \mathbf{k}-\overline{\mathbf{v}}_{1}\right)+(\Delta \rho / 2)(\hat{\mathrm{b}} \beta \omega)^{2}\left(\overline{\mathbf{v}}_{2} \cdot \nabla\right)\left(\bar{T} \mathbf{k}-\overline{\mathbf{v}}_{2}\right)$

Here $\mathbf{u}, \rho_{0}$ are the fluid convection velocity and density of the base fluid; $t$ is the time; $p, \mu$ are excess pressure and the dynamic viscosity; $n, \theta$ are concentration of active particles and average volume of an active particle; $\Delta \rho=\rho_{\mathrm{c}}-\rho_{0}, \beta$ are the density difference between particles and base fluid and the coefficient of volumetric thermal expansion (of base fluid); $\mathbf{g}, \hat{b}$ are gravity vector and amplitude of vertical oscillation; $\omega, \mathbf{k}$ are angular frequency of oscillation and the vertically upward unit vector in z-direction; $q_{c}, \hat{\mathbf{p}}$ denote swimming speed of active particles and the vector indicating the swimming path; $D_{c}, c_{p}$ are diffusivity of active swimmers and the specific heat of fluid medium; and $\kappa$ is the thermal conductivity. High-frequency oscillations with uniform/non-uniform thermal fields are known to exert a thermo convective vibrational flow in which all the physical variables are sought to be separated into two distinct components $[14,16]$. One part fluctuates quickly with time while the other one changes slowly with time. In order to obtain the theoretical forms of time averaged equations, certain approximations have to be validated [36]. The frequency of oscillation is considerably high which makes the period of vibration (over a specified period $\tau=2 \pi / \omega)$ small compared to all the $\partial \bar{n} / \partial t=-\operatorname{div}\left[\bar{n} \overline{\mathbf{u}}+\bar{n} q_{c} \hat{\mathbf{p}}-D_{c} \nabla \bar{n}\right]$

$c_{p} \rho_{0}[\partial \bar{T} / \partial t+(\overline{\mathbf{u}} \cdot \nabla) \bar{T}]=\kappa \nabla^{2} \bar{T}$

The quantities with over bar refer to the mean variables. Here, the last two terms in Eq. (6) stands for the averaged volumetric and convective forces of vibrational character [17]. The vectors $\overline{\mathbf{v}}_{1}$ and $\overline{\mathbf{v}}_{2}$ are the solenoidal component of the vectors quantities $\bar{n} \mathbf{k}$ and $\bar{T} \mathbf{k}$, respectively, and are supposed to satisfy the Helmholtz decomposition [37]:

$\operatorname{curl} \overline{\mathbf{v}}_{1}=\operatorname{grad} \bar{n} \wedge \mathbf{k}, \quad \operatorname{div} \overline{\mathbf{v}}_{1}=0$,

$\operatorname{curl} \overline{\mathbf{v}}_{2}=\operatorname{grad} \bar{T} \wedge \mathbf{k}, \quad \operatorname{div} \overline{\mathbf{v}}_{2}=0$

For a rigid-rigid suspension the conditions are $\overline{\mathbf{u}}=\mathbf{0}$, $\mathbf{j} \cdot \mathbf{k}=\overline{\mathbf{v}}_{1} \cdot \mathbf{k}=\overline{\mathbf{v}}_{2} \cdot \mathbf{k}=0, \bar{T}=T_{0}+\Delta T$ at the lower surface $(z=0)$ and $\overline{\mathbf{u}}=\mathbf{0}, \mathbf{j} \cdot \mathbf{k}=\overline{\mathbf{v}}_{1} \cdot \mathbf{k}=\overline{\mathbf{v}}_{2} \cdot \mathbf{k}=0$, $\bar{T}=T_{0}$ on the upper surface $(z=d)$. Also for a rigid-free suspension boundary conditions at the top layer are $\left[\partial^{2} / \partial z^{2}\right](\overline{\mathbf{u}} \cdot \mathbf{k})=0, \overline{\mathbf{u}} \cdot \mathbf{k}=0, \mathbf{j} \cdot \mathbf{k}=\overline{\mathbf{v}}_{1} \cdot \mathbf{k}=\overline{\mathbf{v}}_{2} \cdot \mathbf{k}=0$, $\bar{T}=T_{0}$. The quantity $\mathbf{j}\left(=n \mathbf{u}+n q_{c} \hat{\mathbf{p}}-D_{c} \nabla n\right)$ represents total flux of active particles across the surface which is exerted due to advection, self-propulsion and diffusion 
[2]. The base state is motionless and is obtained as follows

$[11,24]$ :

$\left.\begin{array}{l}\overline{\mathbf{u}}_{o}=\mathbf{0}, \quad \hat{\mathbf{p}}_{o}=\mathbf{k}, \quad \bar{n}_{o}(z)=v \exp \left(q_{c} z / D_{c}\right) \Rightarrow v=\bar{n}_{a v} P e /(\exp (P e)-1), \quad P e=q_{c} d / D_{c^{\prime}} \\ \bar{T}_{o}(z)=T_{0}+\Delta T[1-(z / d)], \quad \overline{\mathbf{v}}_{1_{o}}=\mathbf{0}, \quad \overline{\mathbf{v}}_{2_{o}}=\mathbf{0}, \\ \bar{p}_{o}(z)=p_{0}+v g \theta \Delta \rho\left(D_{c} / q_{c}\right)\left[\exp (P e)-\exp \left(q_{c} z / D_{c}\right)\right]-\mathrm{g} \rho_{0} \beta \Delta T\left[(d-z)-\left(d^{2}-z^{2}\right) / 2 d\right]\end{array}\right\}$

The subscript ' $o$ ' refers to the fundamental state. Here $\bar{n}_{a v}$ refers to the average cell concentration, constant $v$ defines the basic cell concentration at the bottom surface and $P e$ is the Péclet number [20-22]. In order to accomplish linear stability results, the small perturbations are applied to the basic variables. The coupled system of equations for the perturbations $\overline{\mathbf{u}}^{*}=\left(u_{1}, u_{2}, u_{3}\right), \overline{\mathbf{v}}_{1}^{*}=\left(v_{11}, v_{12}, v_{13}\right), \overline{\mathbf{v}}_{2}^{*}=\left(v_{21}, v_{22}, v_{23}\right), \overline{\boldsymbol{p}}^{*}, \hat{\mathbf{p}}^{*}, T^{*}$ and $\bar{n}^{*}$, which refers to the perturbations to fluid velocity, vibrogenic body force, vibrogenic convective force, pressure, average swimming direction, temperature and number density of the gyrotactic swimmers, respectively, are rewritten as:

$\nabla \cdot \overline{\mathbf{u}}^{*}=0$

$\rho_{0}\left(\partial \overline{\mathbf{u}}^{*} / \partial t\right)=-\nabla \bar{p}^{*}+\mu \nabla^{2} \overline{\mathbf{u}}^{*}+\mathbf{g}\left(\bar{n}^{*} \theta \Delta \rho-\rho_{0} \beta \bar{T}^{*}\right)+\left(\rho_{0} / 2\right)\left[\left(\Delta \rho / \rho_{0}\right) \hat{\mathrm{b}} \theta \omega\right]^{2}\left(\overline{\mathbf{v}}_{1}^{*} \cdot \nabla\right)\left(\bar{n}^{*} \mathbf{k}-\overline{\mathbf{v}}_{1}^{*}\right)+(\Delta \rho / 2)(\hat{\mathrm{b}} \beta \omega)^{2}\left(\overline{\mathbf{v}}_{2}^{*} \cdot \nabla\right)\left(\bar{T}^{*} \mathbf{k}-\overline{\mathbf{v}}_{2}^{*}\right)$

$\partial \bar{n}^{*} / \partial t=-\operatorname{div}\left[\bar{n}_{o}\left(\overline{\mathbf{u}}^{*}+q_{c} \hat{\mathbf{p}}^{*}\right)+\bar{n}^{*} q_{c} \mathbf{k}-D_{c} \nabla \bar{n}^{*}\right]$

$c_{p} \rho_{0}\left[\partial \bar{T}^{*} / \partial t-u_{3}\left(\Delta \bar{T}^{*} / d\right)\right]=\kappa \nabla^{2} \bar{T}^{*}$

$\operatorname{curl} \overline{\mathbf{v}}_{1}^{*}=\operatorname{grad} \bar{n}^{*} \wedge \mathbf{k}, \quad \operatorname{curl} \overline{\mathbf{v}}_{2}^{*}=\operatorname{grad} \bar{T}^{*} \wedge \mathbf{k}$

Applying k · curl curl and $\mathbf{k} \cdot$ curl on Eqs. (12) and (15), respectively:
$\left.\begin{array}{l}\hat{\mathbf{p}}^{*}=\mathrm{B}(\eta,-\xi, 0), \eta=\left(1+\alpha_{0}\right) \partial u_{1} / \partial z-\left(1-\alpha_{0}\right) \partial u_{3} / \partial x \\ \mathrm{~B}=\alpha_{\perp} \mu / 2 h \rho_{0} g, \xi=\left(1-\alpha_{0}\right) \partial u_{3} / \partial y-\left(1+\alpha_{0}\right) \partial u_{2} / \partial z, \\ \alpha_{0}=a^{2}-b^{2} / a^{2}+b^{2}\end{array}\right\}$

The parameters $\mathrm{B}, \alpha_{\perp}, h, \alpha_{0}, a$ and $b$ are having their standard meanings and are utilized by authors in previous studies [2, 20-22]. Using Eq. (18) in to Eq. (13):

$\partial \bar{n}^{*} / \partial t=-\left(\partial \bar{n}_{o} / \partial z\right) u_{3}-\left(\partial \bar{n}^{*} / \partial z\right) q_{c}+q_{c} \mathrm{~B}\left[\left(1-\alpha_{0}\right) \nabla^{1} u_{3}\right.$ $\left.+\left(1+\alpha_{0}\right) \partial^{2} u_{3} / \partial z^{2}\right] \bar{n}_{o}+D_{c} \nabla^{2} \bar{n}^{*}$

Transforming the disturbances into normal modes: $\left(u_{3}, \bar{n}^{*}, \bar{T}^{*}, v_{13}, v_{23}\right)=\left[U_{3}, N_{3}, \Theta, V_{13}, V_{23}\right](z) f(x, y) \exp \left(\sigma_{s} t\right)$

Here, $\left(\nabla^{1}+m^{2}\right) f(x, y)=0$, and ' $m$ ' is the horizontal wave number [38]. Utilizing Eq. (20), non-dimensionalized equations are:

$D^{4} U_{3}^{\diamond}-\left(2 a^{\diamond^{2}}+\sigma\right) D^{2} U_{3}^{\diamond}+\left(a^{\diamond^{4}}+a^{\diamond^{2}} \sigma\right) U_{3}^{\diamond}$

$+a^{\diamond^{2}}\left[\tilde{R} b\left(N_{3}^{\diamond}-\left(\Theta^{\diamond} / \mho\right)\right)-R v \exp \left(z^{\diamond} P e\right) V_{13}^{\diamond}+R v_{b} V_{23}^{\diamond}\right]=0$

$\rho_{0}(\partial / \partial t)\left(\nabla^{2} u_{3}\right)=\mu\left[2\left(\partial^{4} u_{3} / \partial x^{2} \partial y^{2}+\partial^{4} u_{3} / \partial y^{2} \partial z^{2}+\partial^{4} u_{3} / \partial z^{2} \partial x^{2}\right)+\partial^{4} u_{3} / \partial x^{4}+\partial^{4} u_{3} / \partial y^{4}+\partial^{4} u_{3} / \partial z^{4}\right]+g\left(\rho_{0} \beta \nabla^{1} \bar{T}^{*}-\theta \Delta \rho \nabla^{1} \bar{n}^{*}\right)$

$+\left(\rho_{0} / 2\right)\left\{\left(\Delta \rho / \rho_{0}\right) \hat{\mathrm{b}} \theta \omega\right\}^{2}\left(\partial \bar{n}_{0} / \partial z\right) \nabla^{1}\left(v_{13}\right)+(\Delta \rho / 2)(\hat{\mathrm{b}} \beta \omega)^{2}\left(\partial \bar{T}_{0} / \partial z\right) \nabla^{1}\left(v_{23}\right)$

$\nabla^{2} v_{13}=\nabla^{1} \bar{n}^{*}, \quad \nabla^{2} v_{23}=\nabla^{1} \bar{T}^{*}$

Here, $\nabla^{1}=\partial^{2} / \partial x^{2}+\partial^{2} / \partial y^{2}$ and $\hat{\mathbf{p}}^{*}$ is the mean swimming direction of gyrotactic swimmers. It is understood that all the cells are symmetrical about $\hat{\mathbf{p}}^{*}$ and possesses the changeless velocity $q_{c} \hat{\mathbf{p}}^{*}$. For the unit vector $\hat{\mathbf{p}}^{*}$, the following relations along with their theoretical explanations are available in literature [2]:

$$
\begin{gathered}
D^{2} N_{3}^{\diamond}-P e D N_{3}^{\diamond}-\left(a^{\diamond^{2}}+\sigma L e \operatorname{Pr}\right) N_{3}^{\diamond}+\left[\left(1+\alpha_{0}\right) G D^{2}\right. \\
\left.-\left(1+\left(1-\alpha_{0}\right) G a^{\diamond^{2}}\right)\right] U_{3}^{\diamond} \exp \left(z^{\diamond} P e\right)=0
\end{gathered}
$$

$D^{2} \Theta^{\diamond}-\left(a^{\diamond^{2}}+\sigma \operatorname{Pr}\right) \Theta^{\diamond}+(\mho R a / \tilde{R} b) U_{3}^{\diamond}=0$

$$
D^{2} V_{13}^{\diamond}+a^{\diamond^{2}}\left(N_{3}^{\diamond}-V_{13}^{\diamond}\right)=0, \quad D^{2} V_{23}^{\diamond}+a^{\diamond^{2}}\left(\Theta^{\diamond}-V_{23}^{\diamond}\right)=0
$$


The dimensionless quantities and parameters used above are defined as follows:

$$
\begin{array}{ll}
\text { at } z^{\diamond}=0, \quad 1 ; & U_{3}^{\diamond}=0, \quad D U_{3}^{\diamond}=0, \\
P e N_{3}^{\diamond}=D N_{3}^{\diamond}, \quad \Theta^{\diamond}=0, \quad V_{13}^{\diamond}=0, \quad V_{23}^{\diamond}=0
\end{array}
$$

$\left.z^{\diamond}=z / d, \quad a^{\diamond}=m d, \quad U_{3}^{\diamond}=\nu \theta q_{c} d^{2} U_{3} / D_{c^{\prime}}^{2} \quad \sigma=\sigma_{s} d^{2} \rho_{0} / \mu, \quad \operatorname{Pr}=\mu c_{p} / \kappa, \quad L e=\kappa / D_{c} \rho_{0} c_{p^{\prime}},\right\}$

$\left.V_{13}^{\diamond}=V_{13} \theta, \quad V_{23}^{\diamond}=V_{23} \beta, \quad G=B D_{c} / d^{2}, \quad N^{\diamond}=N \theta, \quad \Theta^{\diamond}=\Theta \beta, \quad \mho=\Delta \rho / \rho_{0}, \quad, \quad D \equiv \partial / \partial z^{\diamond}\right\}$

The suitable test solutions satisfying the boundaries

Here, $R b=\left(g \nu \theta \Delta \rho d^{3} / \mu D_{c}\right)$ is the bioconvection Rayleigh (27) are chosen as follows:

number, $\mho$ is the density measure of gyrotactic swimmers,

(27) are chosen as follows:

$\left.\begin{array}{l}\left(U_{3}^{\diamond}\right)_{1}=z^{\diamond^{2}}\left(1-z^{\diamond}\right)^{2}, \quad\left(N_{3}^{\diamond}\right)_{1}=2-P e\left(1-2 z^{\diamond}\right)-(P e)^{2}\left(z^{\diamond}-z^{\diamond^{2}}\right) \\ \left(V_{23}^{\diamond}\right)_{1}=\left(z^{\diamond}-z^{\diamond^{2}}\right), \quad\left(\Theta^{\diamond}\right)_{1}=z^{\diamond}\left(1-z^{\diamond}\right), \quad\left(V_{13}^{\diamond}\right)_{1}=\left(z^{\diamond}-z^{\diamond^{2}}\right)\end{array}\right\}$

Pr is the Prandtl number, $G$ is the gyrotactic number, $L$ is the Lewis number, $R a=\rho_{0}^{2} d^{3} c_{p} g \beta \Delta T / \mu \kappa$ is the thermal Rayleigh number, $R v=\rho_{0}\left\{\theta \hat{b} \omega v q_{c} d^{2}\left(\Delta \rho / \rho_{0}\right)\right\}^{2} / 2 \mu D_{c}^{3}$ is
Replacing the above test functions [Eq. (28)] into Eqs. (21)-(24) and applying the one term Galerkin scheme the eigen value relation takes the form:

$$
\begin{aligned}
& 28\left(10+a^{\diamond^{2}}\right)\left(10+a^{\diamond^{2}}+\sigma \operatorname{Pr}\right)\left[\left(a^{\diamond^{4}}+24 a^{\diamond^{2}}+504\right)+\sigma\left(12+a^{\diamond^{2}}\right)\right]\left[\left(120-10(P e)^{2}+(P e)^{4}\right)\left(\sigma P r L e+a^{\diamond^{2}}\right)+10(P e)^{4}\right] \\
& =\left[1260 a^{\diamond^{2}} \chi\left(10+a^{\diamond^{2}}\right)\left(28-3(P e)^{2}\right)\left(10+a^{\diamond^{2}}+\sigma \operatorname{Pr}\right)\right] \tilde{R} b+\left[529200 \chi \chi_{3} a^{\diamond^{4}}\left(10-(P e)^{2}\right)\left(10+a^{\diamond^{2}}+\sigma \operatorname{Pr}\right)\right] R v \\
& +27 a^{\diamond^{2}}\left[\left(\sigma \operatorname{Pr} L e+a^{\diamond^{2}}\right)\left(120-10(P e)^{2}+(P e)^{4}\right)+10(P e)^{4}\right]\left[\left(10+a^{\diamond^{2}}\right) R a-a^{\diamond^{2}} R a_{v b}\right]
\end{aligned}
$$

the vibrational Rayleigh number, $\sigma$ is the growth rate and $R v_{b}=\Delta \rho q_{c} \nu \theta \beta d^{3} \Delta T\{\hat{b} \omega\}^{2} / 2 \mu D_{c}^{2}$ is the thermo-vibrational parameter. Here, $\tilde{R} b=P e R b$ is the modified (analogues) bioconvection Rayleigh number. To obtain the solutions of the Eqs. (21)-(24), we apply the Galerkin method of weighted residuals [39]. For the trial functions satisfying the dimensionless boundaries the following expressions are formed:

$\left.U_{3}^{\diamond}=\sum_{j=1}^{M} A_{j}\left(U_{3}^{\diamond}\right)_{j^{\prime}} \quad N_{3}^{\diamond}=\sum_{j=1}^{M} B_{j}\left(N_{3}^{\diamond}\right)_{j^{\prime}} \quad \Theta^{\diamond}=\sum_{j=1}^{M} C_{j}\left(\Theta^{\diamond}\right)_{j^{\prime}} \quad V_{13}^{\diamond}=\sum_{j=1}^{M} D_{j}\left(V_{13}^{\diamond}\right)_{j^{\prime}} \quad V_{23}^{\diamond}=\sum_{j=1}^{M} E_{j}\left(V_{23}^{\diamond}\right)_{j}\right\}$

Substituting Eq. (26) into system of Eqs. (21)-(24) and applying the standard procedure we get a coupled system of $5 M$ equations in $5 M$ variables $A_{j}, B_{j}, C_{j}, D_{j}$ and $E_{j}$. A non trivial solution of this homogeneous system of equations leads to an eigenvalue equation.

\subsection{Case I: rigid upper and rigid bottom walls}

For this case the top and lower surfaces are considered to be rigid. The single term Galerkin scheme is invoked and the dimensionless boundaries are given as follows
Here, $R a_{v b}=\left(\delta R a R v_{b} / \tilde{R} b\right)$ and $\chi$ is a functions of $\chi_{1}, \chi_{2}, a^{\diamond}, \alpha_{0}, G$ and $\chi_{1}, \chi_{2}, \chi_{3}$ are functions of $P e$ only. These are explicated in Appendix 1 [Eqs. (51)-(54)]. At the onset of oscillatory convection substituting $\sigma=i \omega$ into Eq. (29), where $\omega$ is the dimensionless frequency (real) of vibrational amplitude. Rearranging and rewriting Eq. (29) the stability boundary is then given by:

$$
\begin{aligned}
& \left(\alpha_{1}+i \omega \beta_{1}\right) \tilde{R} b+\left(\alpha_{2}+i \omega \beta_{2}\right) R a+\left(\alpha_{3}+i \omega \beta_{3}\right) R v \\
& \quad-\left(\alpha_{4}+i \omega \beta_{4}\right) R a_{v b}=\left(\alpha_{5}+i \omega \beta_{5}\right)\left(\alpha_{6}+i \omega \beta_{6}\right)\left(\alpha_{7}+i \omega \beta_{7}\right)
\end{aligned}
$$

The expressions for the symbols $\alpha_{i}, \beta_{i}(i=1,2, \ldots 7)$, are defined in the Appendix 1 [Eqs. (55)-(60)].

\subsubsection{Non-oscillatory convection}

For this case, substituting $\omega=0$ in Eq. (30), it gives 
$\alpha_{1} \tilde{R} b+\alpha_{2} R a+\alpha_{3} R v-\alpha_{4} R a_{v b}=\alpha_{5} \alpha_{6} \alpha_{7}$

The stationary stability boundary is then given by the relation

$\left(\tilde{R} b / R b_{0}+R v / R v_{0}\right)+\left(R a / R a_{0}-R a_{v b} / R a_{v b_{0}}\right)=1$

Here the quantities $\alpha_{5} \alpha_{6} \alpha_{7} / \alpha_{1}=R b_{0}, \alpha_{5} \alpha_{6} \alpha_{7} / \alpha_{2}=R a_{0}$ ， $\alpha_{5} \alpha_{6} \alpha_{7} / \alpha_{3}=R v_{0}$ and $\alpha_{5} \alpha_{6} \alpha_{7} / \alpha_{4}=R a_{v b_{0}}$ are the new denominations introduced. The critical bioconvection negatively geotactic swimmers. These equations agree completely with the result obtained by researchers [20, 24].

\subsubsection{Oscillatory convection}

For oscillatory convection the imaginary and real components of Eq. (30) are considered for the elimination of frequency of vibration which gives:

$$
\begin{aligned}
\omega^{2} & =\left[\left(\alpha_{5} \alpha_{6} \beta_{7}+\alpha_{5} \alpha_{7} \beta_{6}+\alpha_{6} \alpha_{7} \beta_{5}\right)-\beta_{1} \tilde{R} b-\beta_{2} R a-\beta_{3} R v+\beta_{4} R a_{v b}\right] / \beta_{5} \beta_{6} \beta_{7} \\
& =\left(\alpha_{5} \alpha_{6} \alpha_{7}-\alpha_{1} \tilde{R} b-\alpha_{2} R a-\alpha_{3} R v+\alpha_{4} R a_{v b}\right) /\left(\beta_{5} \beta_{6} \alpha_{7}+\beta_{6} \beta_{7} \alpha_{5}+\beta_{5} \beta_{7} \alpha_{6}\right)
\end{aligned}
$$

Rayleigh number for the stationary convection is then and the boundary of oscillatory instability is given by:

$\tilde{R} b_{c r}=\operatorname{Min}_{a^{\diamond} \geq 0}\left[\left(R b_{0} / 2\right) \sqrt{\left\{R a / R a_{0}+R v / R v_{0}-1\right\}^{2}+\left(4 \delta R a R v_{b} / R b_{0} R a_{v b_{0}}\right)}-\left(R b_{0} / 2\right)\left(R a / R a_{0}+R v / R v_{0}-1\right)\right]$

In the limiting case when $P e$ tends to zero, $G$ tends to zero and in the absence of vibration effect Eq. (32) collapse to:

$\tilde{R} b / 720+R a / 1750=1$

Equation (34) is the same expression as reported by Kuznetsov for the case of thermal suspension of gyrotactic swimmers in the non-existence of the vertical vibration [11]. The limiting values for $R b_{0}, R a_{0}, R v_{0}$ and $R a_{v b_{0}}$ as $P e \rightarrow 0$ and $G \rightarrow 0$ are given in Appendix 1 [Eqs. (61)-(62)]. Also in the absence of thermal gradient Eq. (32) can be re-caste as:

$\tilde{R} b / R b_{0}+R v / R v_{0}=1, \quad$ and $\quad \tilde{R} b_{c r}=\operatorname{Min}_{a^{\diamond} \geq 0}\left[10\left(a^{\diamond^{4}}+24 a^{\diamond^{2}}+504\right) / 7\right]$

From the second equation in Eq. (35), it is obtained that $\tilde{R} b_{c r}=720$, for the corresponding value of critical wave number $a_{c r}^{\diamond}=0$ (in the absentia of vibrational impact). These values match exactly with those reported by Sparrow and co-workers for the case of rigid-rigid surfaces [40]. Equations (35) give the stationary instability boundary and the critical strength of bioconvection for

$$
\begin{aligned}
& \left(\alpha_{7} \beta_{1} \beta_{5} \beta_{6}+\alpha_{6} \beta_{1} \beta_{5} \beta_{7}+\alpha_{5} \beta_{1} \beta_{6} \beta_{7}-\alpha_{1} \beta_{5} \beta_{6} \beta_{7}\right) \tilde{R} b \\
& +\left(\alpha_{7} \beta_{2} \beta_{5} \beta_{6}+\alpha_{6} \beta_{2} \beta_{5} \beta_{7}+\alpha_{5} \beta_{2} \beta_{6} \beta_{7}-\alpha_{2} \beta_{5} \beta_{6} \beta_{7}\right) R a \\
& +\left(\alpha_{7} \beta_{3} \beta_{5} \beta_{6}+\alpha_{6} \beta_{3} \beta_{5} \beta_{7}+\alpha_{5} \beta_{3} \beta_{6} \beta_{7}-\alpha_{3} \beta_{5} \beta_{6} \beta_{7}\right) R v \\
& +\left(\alpha_{4} \beta_{5} \beta_{6} \beta_{7}-\alpha_{7} \beta_{4} \beta_{5} \beta_{6}-\alpha_{6} \beta_{4} \beta_{5} \beta_{7}-\alpha_{5} \beta_{4} \beta_{6} \beta_{7}\right) R a_{v b} \\
& =\left(\alpha_{5} \alpha_{6} \beta_{7}+\alpha_{5} \alpha_{7} \beta_{6}+\alpha_{6} \alpha_{7} \beta_{5}\right)\left(\beta_{5} \beta_{6} \alpha_{7}+\beta_{5} \beta_{7} \alpha_{6}+\beta_{6} \beta_{7} \alpha_{5}\right) \\
& \quad-\left(\alpha_{5} \alpha_{6} \alpha_{7} \beta_{5} \beta_{6} \beta_{7}\right)
\end{aligned}
$$

Clearly, in order to possesses a real $\omega(\omega \neq 0)$, the thermal stratification and bioconvection strength must be opposite in nature. Since a negative $\tilde{R} b$ is meaningless physically, therefore in order to have a possible overstability $R a$ must be negative. To simplify Eq. (37), setting the

$$
\begin{aligned}
\omega^{2}=[1 & \left.-\left(\tilde{R} b / R b_{0}+R v / R v_{0}\right)-\left(R a / R a_{0}-R a_{v b} / R a_{v b_{0}}\right)\right] /\left(\Lambda_{1} \Lambda_{2}+\Lambda_{2} \Lambda_{5}+\Lambda_{1} \Lambda_{5}\right) \\
= & {\left[\left(\Lambda_{1}+\Lambda_{2}+\Lambda_{5}\right)-\Lambda_{1}\left(\tilde{R} b / R b_{0}+R v / R v_{0}\right)-\Lambda_{2}\left(R a / R a_{0}-R a_{v b} / R a_{v b_{0}}\right)\right] /\left(\Lambda_{1} \Lambda_{2} \Lambda_{5}\right) }
\end{aligned}
$$

In the limiting case when $P e \rightarrow 0$ and $G \rightarrow 0$, values $\Lambda_{i}(i=1,2, \ldots 7)$ are defined in Appendix 1 [Eq. (63)]. 


\subsection{Case II: rigid lower and stress-free upper surfaces}

In this case, the lower surface satisfies the no-slip condition and the upper wall is considered to be stress free. The conditions for non-dimensionalized lower wall are: $\alpha_{15} \alpha_{16} \alpha_{17}=\alpha_{11} \tilde{R} b+\alpha_{12} R a+\alpha_{13} R v-\alpha_{14} R a_{v b}$

Then the non-oscillatory instability boundary may be written as:

$\left(\tilde{R} b / R b_{1}+R v / R v_{1}\right)+\left(R a / R a_{1}-R a_{v b} / R a_{v b_{1}}\right)=1$

at $\quad z^{\diamond}=0 ; \quad U_{3}^{\diamond}=0, \quad D U_{3}^{\diamond}=0, \quad P e N_{3}^{\diamond}=D N_{3}^{\diamond}, \quad \Theta^{\diamond}=0, \quad V_{13}^{\diamond}=0, \quad V_{23}^{\diamond}=0$

When the upper boundary is stress free, the second condition in Eq. (40) is removed and put back with $D^{2} U_{3}^{\diamond}=0$ at $z^{\diamond}=1$. The preferable test functions satisfying Eq. (40) are:

The new denominations being used are $\alpha_{15} \alpha_{16} \alpha_{17} / \alpha_{11}=R b_{1} \quad, \quad \alpha_{15} \alpha_{16} \alpha_{17} / \alpha_{12}=R a_{1}$ $\alpha_{15} \alpha_{16} \alpha_{17} / \alpha_{13}=R v_{1}$ and $\alpha_{15} \alpha_{16} \alpha_{17} / \alpha_{14}=R a_{v b_{1}}$. The asso-

$\left.\begin{array}{l}\left(U_{3}^{\diamond}\right)_{1}=z^{\diamond^{2}}\left(3-2 z^{\diamond}\right)\left(1-z^{\diamond}\right), \quad\left(N_{3}^{\diamond}\right)_{1}=2-\left(z^{\diamond}-z^{\diamond^{2}}\right)(P e)^{2}-\left(1-2 z^{\diamond}\right) P e \\ \left(V_{13}^{\diamond}\right)_{1}=z^{\diamond}\left(1-z^{\diamond}\right), \quad\left(V_{23}^{\diamond}\right)_{1}=\left(z^{\diamond}-z^{\diamond^{2}}\right), \quad\left(\Theta^{\diamond}\right)_{1}=z^{\diamond}\left(1-z^{\diamond}\right)\end{array}\right\}$

Putting, Eq. (41) into Eqs. (21)-(24) and invoking the standard Galerkin scheme the characteristic equation takes the form: ciated critical bioconvection Rayleigh number with the monotonic convection is then given by:

$$
\begin{aligned}
& 28\left(10+a^{\diamond^{2}}\right)\left(10+a^{\vartheta^{2}}+\sigma \operatorname{Pr}\right)\left[\left(a^{\diamond^{2}}+\sigma \operatorname{Pr} L e\right)\left(120-10(P e)^{2}+(P e)^{4}\right)+10(P e)^{4}\right] \\
& \times\left[19\left(a^{\diamond^{4}}+\sigma a^{\diamond^{2}}\right)+216\left(2 a^{\diamond^{2}}+\sigma\right)+4536\right]=\left[1260 a^{\diamond^{2}} \zeta\left(10+a^{\diamond^{2}}\right)^{2}\left(126+7 P e-13(P e)^{2}\right)\left(10+a^{\diamond^{2}}+\sigma \operatorname{Pr}\right)\right] \tilde{R} b \\
& +\left[529200 \zeta \zeta_{3} a^{\diamond^{4}}\left(10-(P e)^{2}\right)\left(10+a^{\diamond^{2}}+\sigma \operatorname{Pr}\right)\right] R v+507 a^{\diamond^{2}}\left[\left(\sigma \operatorname{Pr} L e+a^{\diamond^{2}}\right)\left(120-10(P e)^{2}+(P e)^{4}\right)+10(P e)^{4}\right] \\
& {\left[\left(10+a^{\diamond^{2}}\right) R a-a^{\diamond^{2}} R a_{v b}\right]}
\end{aligned}
$$

Quantity, $R a_{v b}=\left(\delta R a R v_{b} / \tilde{R} b\right)$ is same as defined for the rigid-rigid case and the symbol $\zeta$ is a functions of $\zeta_{1}, \zeta_{2}, G, \alpha_{0}$ and the wave number $a^{\diamond}$. Functions $\zeta_{1}, \zeta_{2}$ and $\zeta_{3}$ are depending on Pe only and their detailed expressions are given in Appendix 2 [Eqs. (64)-(67)]. At the onset of overstability in the system; analyzing Eq. (42) for $\sigma=i \omega$, ( $\omega$ is real) the equation for the stability boundary may be written as:

$$
\begin{aligned}
\tilde{R} b_{c r}= & \operatorname{Min}_{a^{\diamond} \geq 0}\left[\left(R b_{1} / 2\right)\left\{\left(R a / R a_{1}+R v / R v_{1}-1\right)^{2}+\left(4 \delta R a R v_{b} / R b_{1} R a_{v b_{1}}\right)\right\}^{1 / 2}\right. \\
& \left.-\left(R a / R a_{1}+R v / R v_{1}-1\right)\left(R b_{1} / 2\right)\right]
\end{aligned}
$$

In the situation when there is no vibration in the system, Eq. (45) reduces to $\tilde{R} b / R b_{1}+R a / R a_{1}=1$. In the limiting case when $P e$ tends to zero and when there is no gyrotaxis, this relation yields $\tilde{R} b_{c r}=320$, which is being attained at

$\left(\alpha_{11}+i \omega \beta_{11}\right) \tilde{R} b+\left(\alpha_{12}+i \omega \beta_{12}\right) R a+\left(\alpha_{13}+i \omega \beta_{13}\right) R v-\left(\alpha_{14}+i \omega \beta_{14}\right) R a_{v b}=\left(\alpha_{15}+i \omega \beta_{15}\right)\left(\alpha_{16}+i \omega \beta_{16}\right)\left(\alpha_{17}+i \omega \beta_{17}\right)$

The detail of the symbols $\alpha_{1 i}, \beta_{1 i}(i=1,2, \ldots 7)$, are given in the Appendix 2 [Eqs. (68)-(73)].

\subsubsection{Non-oscillatory convection}

Setting $\omega=0$ in Eq. (43), for the onset of non-oscillatory convection, we obtain: $a_{c r}^{\diamond}=0$. Also for the traditional thermal convection $(R b=0$ i.e., when there are no active swimmers) the reported value for the lower bound of thermal Rayleigh number is $R a_{c r}=1139$ and the respective critical wave number is $a_{c r}^{\diamond}=2.67$. These values match exactly with those achieved in [23]. Thus in a restricted environment (for regular 
thermal convection of binary fluid) the values $R a_{c r}=1139$ and $a_{c r}^{\diamond}=2.67$ are simply $3.48 \%$ bigger than and $0.45 \%$ slighter than the respective accurate values reported for the same quantities $\left(R a_{c r}=1100.65\right.$ and $\left.a_{c r}^{\diamond}=2.682\right)$ [38]. This accomplishes an approximation of the anticipated precision of the single-term Galerkin scheme for the current study. When there is no thermal effect, Eq. (45) collapses to $\tilde{R} b / R b_{1}+R v / R v_{1}=1$. When there is no vibration, the lower bound for the modified bioconvection Rayleigh number is $\tilde{R} b_{c r}=\operatorname{Min}_{a^{\diamond}>0}\left[40\left(19 a^{\diamond^{4}}+432 a^{\diamond^{2}}+4536\right) / 567\right]$. Also in the limiting case, (i.e., when $P e \rightarrow 0$ and $G \rightarrow 0$ ), the estimated values of $R b_{1}, R a_{1}, R v_{1}$ and $R a_{v b_{1}}$ are given in Appendix 2 [Eqs. (74)-(75)].

\subsubsection{Oscillatory convection}

For the onset of overstability in the system the real and imaginary components of Eq. (43) should be considered and the elimination of frequency $\omega$ gives:

\section{Results and discussions}

In this section the variation of typical parameters such as Péclet number, gyrotactic numbers, vibrational Rayleigh number and thermo-vibrational parameter over the instability regions of the oscillatory and stationary modes of convections have been discussed and compared in detail. The results are plotted to study the dependence of the controlling parameters such as modified critical bioconvection Rayleigh number $\tilde{R} b_{c r}$, thermal Rayleigh number $R a$ and the critical wave number $a_{c r}^{\diamond}$ for the varying fixed values cell eccentricity $\alpha_{0}$, gyrotactic number $G$, Péclet number $P e$, vibrational Rayleigh number $R v$ and thermo-vibrational parameter $R v_{b}$. Especially, for the swimmers like Chlamydomonas nivalis, the feasible and estimated ranges of these parameters exist in the literature and are utilized by numerous authors in their respective studies $[2,11,21,22,24]$. The bioconvection Rayleigh number $\tilde{R} b_{c r}$ is the dimensionless quantity which

$$
\begin{aligned}
\omega^{2} & =\left(\alpha_{15} \alpha_{16} \alpha_{17}-\alpha_{11} \tilde{R} b-\alpha_{12} R a-\alpha_{13} R v+\alpha_{14} R a_{v b}\right) /\left(\beta_{15} \beta_{16} \alpha_{17}+\beta_{16} \beta_{17} \alpha_{15}+\beta_{15} \beta_{17} \alpha_{16}\right) \\
& =\left[\left(\alpha_{15} \alpha_{16} \beta_{17}+\alpha_{15} \alpha_{17} \beta_{16}+\alpha_{16} \alpha_{17} \beta_{15}\right)-\beta_{11} \tilde{R} b-\beta_{12} R a-\beta_{13} R v+\beta_{14} R a_{v b}\right] / \beta_{15} \beta_{16} \beta_{17}
\end{aligned}
$$

The boundary for the oscillatory convection becomes

$$
\begin{aligned}
& \left(\alpha_{17} \beta_{11} \beta_{15} \beta_{16}+\alpha_{16} \beta_{11} \beta_{15} \beta_{17}+\alpha_{15} \beta_{11} \beta_{16} \beta_{17}-\alpha_{11} \beta_{15} \beta_{16} \beta_{17}\right) \tilde{R} b \\
& +\left(\alpha_{17} \beta_{12} \beta_{15} \beta_{16}+\alpha_{16} \beta_{12} \beta_{15} \beta_{17}+\alpha_{15} \beta_{12} \beta_{16} \beta_{17}-\alpha_{12} \beta_{15} \beta_{16} \beta_{17}\right) R a \\
& +\left(\alpha_{17} \beta_{13} \beta_{15} \beta_{16}+\alpha_{16} \beta_{13} \beta_{15} \beta_{17}+\alpha_{15} \beta_{13} \beta_{16} \beta_{17}-\alpha_{13} \beta_{15} \beta_{16} \beta_{17}\right) R v \\
& +\left(\alpha_{14} \beta_{15} \beta_{16} \beta_{17}-\alpha_{17} \beta_{14} \beta_{15} \beta_{16}-\alpha_{16} \beta_{14} \beta_{15} \beta_{17}-\alpha_{15} \beta_{14} \beta_{16} \beta_{17}\right) R a_{v b} \\
& =\left(\alpha_{15} \alpha_{16} \beta_{17}+\alpha_{15} \alpha_{17} \beta_{16}+\alpha_{16} \alpha_{17} \beta_{15}\right)\left(\beta_{15} \beta_{16} \alpha_{17}+\beta_{15} \beta_{17} \alpha_{16}+\beta_{16} \beta_{17} \alpha_{15}\right) \\
& \quad-\left(\alpha_{15} \alpha_{16} \alpha_{17} \beta_{15} \beta_{16} \beta_{17}\right)
\end{aligned}
$$

To simplify above equation, introducing the new notations $\Lambda_{1 i}=\beta_{1 i} / \alpha_{1 i},(i=1,2, \ldots 7)$ and using the identities $\Lambda_{11}=\Lambda_{13}=\Lambda_{15}$ and $\Lambda_{12}=\Lambda_{14}=\Lambda_{17}$. Then the overstability boundary becomes characterizes and controls the stability criterion in the suspension. Increase or decrease in its magnitudes leads to a stabilizing or destabilizing behavior of the suspension. At a specific negative price of $R a$, the $\tilde{R} b_{c r}$ in stationary mode surpasses the corresponding $\tilde{R} b_{c r}$ in oscillatory mode and thus the sufficiently large negative $R a$ leads to the occurrence of overstability [20].

In Fig. 1a-c; the simultaneous critical values of $\tilde{R} b_{c r}$ corresponding to the thermal variation are plotted for oscillatory and non-oscillatory modes of convection, when the boundaries of the considered layer are rigid-rigid. We show the results of calculation for the representative values $\operatorname{Pr}=7, \alpha_{0}=0.2$ and $L e=0.33$ of the parameters [21,

$$
\begin{aligned}
& \Lambda_{11}\left(1 / \Lambda_{12}+1 / \Lambda_{16}\right)\left(\tilde{R} b / R b_{1}+R v / R v_{1}\right)+\Lambda_{12}\left(1 / \Lambda_{11}+1 / \Lambda_{16}\right)\left(R a / R a_{1}-R a_{v b} / R a_{v b_{1}}\right) \\
& \quad=\left(\Lambda_{11}+\Lambda_{12}+\Lambda_{16}\right)\left(1 / \Lambda_{11}+1 / \Lambda_{12}+1 / \Lambda_{16}\right)-1
\end{aligned}
$$

and the vibrational frequency is given by:

22]. Growing the thermal effect across the layer it is dis-

$$
\begin{aligned}
\omega^{2}= & =\left[\left(\Lambda_{11}+\Lambda_{12}+\Lambda_{16}\right)-\Lambda_{11}\left(\tilde{R} b / R b_{1}+R v / R v_{1}\right)-\Lambda_{12}\left(R a / R a_{1}-R a_{v b} / R a_{v b_{1}}\right)\right] /\left(\Lambda_{11} \Lambda_{12} \Lambda_{16}\right) \\
& =\left[1-\left(\tilde{R} b / R b_{1}+R v / R v_{1}\right)-\left(R a / R a_{1}-R a_{v b} / R a_{v b_{1}}\right)\right] /\left(\Lambda_{11} \Lambda_{12}+\Lambda_{12} \Lambda_{16}+\Lambda_{11} \Lambda_{16}\right)
\end{aligned}
$$

For the limiting case, when $G \rightarrow 0$ and $P e \rightarrow 0$, the values of $\Lambda_{1 i}(i=1,2, \ldots 7)$ are specified in Appendix 2 [Eq. (76)]. played that $\tilde{R} b_{c r}$ suppresses for both the modes of convection. Therefore, due to presence of vibration, the increase in thermal variation overturns the bioconvection for the 


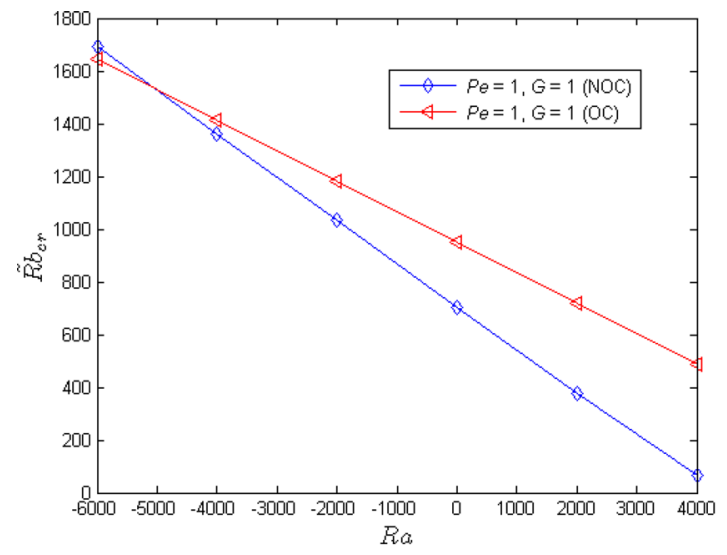

(a)

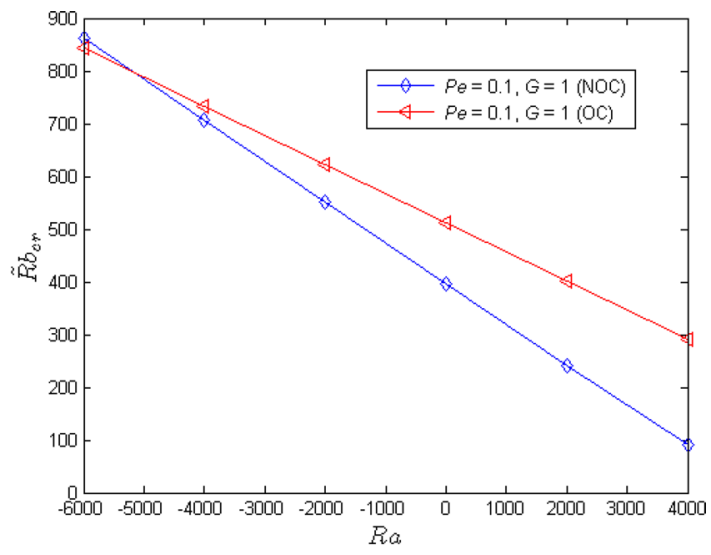

(b)

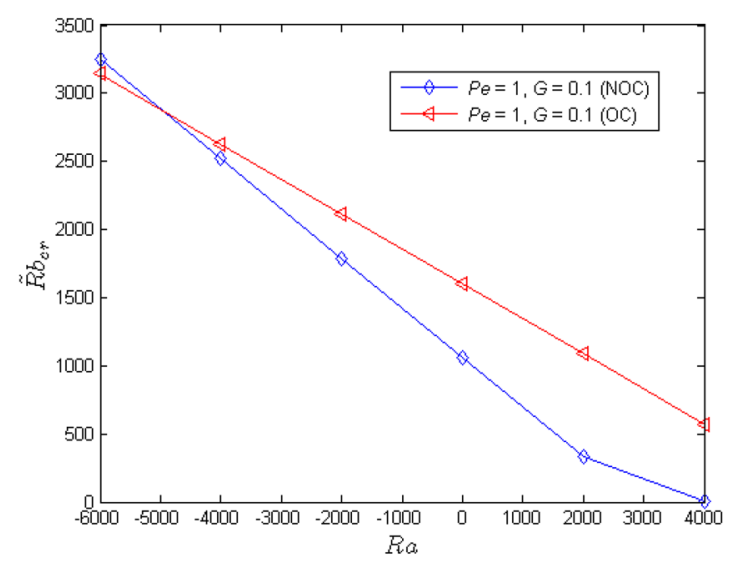

(c)

Fig. 1 The case of rigid upper and lower surfaces: a-c Boundaries of Stationary and oscillatory instability modes of convections in the $\left(R a, \tilde{R} b_{c r}\right)$ plane for the distinct values of Péclet number Pe and Gyrotactic number $G$

vertically oscillated system. For the large Péclet number $(P e=1)$; It is found that, the slope of the $\tilde{R} b_{c r}$ curves is sharper (with higher magnitudes) when the gyrotactic number is smaller $(G=0.1$ ) (Fig. $1 a, c)$. Therefore $\tilde{R} b_{c r}$ is suppressed with increase in gyrotaxis and this demonstrate the unstableness of a uniform gyrotactic suspension for $G>0[2,11]$. Gyrotactic number defines the particles' swimming deviation from the vertical direction hence a growth in gyrotaxis prompts the development in the convective instability [21]. On the other hand; for higher gyrotaxis $(G=1)$, the corresponding values on $\tilde{R} b_{c r}$ axis decreases sharply, for smaller Péclet number $(P e=0.1)$ (Fig. 1a, b). The Péclet number stands for the fractional quantification of the speed of active particles over the speed of bulk fluid motion. Therefore a suspension having moderate active particles is less stable than the system containing the expeditious active swimmers [22].

Figure 2a-c, display the similar study (as Fig. 1) for the case of rigid-free boundaries. It is displayed that for a vertically oscillated system the increment in thermal gradient across the rigid-free layers leads to reduction in intensity of bioconvection. It is found that for higher values of Péclet and gyrotactic numbers the amplitude of $\tilde{R} b_{c r}$ is observed to be smaller than the amplitude of $\tilde{R} b_{c r}$ for the smaller values of these parameters. Therefore a stabilization and destabilization in the system is displayed due to the advancement in Péclet number (Fig. 2a, b) and gyrotactic number (Fig. 2a, c) respectively. The stabilizing nature of Péclet number is induced by the vibrational force which thrusts the mean flow field to work in direction of density homogeneities and the overall fluid circulation grow weaker with increase in oscillations [24,36]. It is demonstrated that the system with stress-free top surface is less stable in comparison to the rigid-rigid suspension [23].

In Fig. 3a-c, dependency of the critical wave number $a_{c r}^{\diamond}$ on the thermal Rayleigh number $R a$ are depicted for the non-oscillatory and oscillatory modes of convections, simultaneously. In these figures the results are shown for rigid-rigid boundaries and graphs are displayed for typical values of gyrotactic and Péclet numbers. In Fig. 4a-c, 
similar studies and results thereof are shown for the both modes of convection for the rigid-free boundary case. For the higher gyrotaxis effect a marginal growth is reported in $a_{c r}^{\diamond}$ as gyrotactic number $G$ increases within the considered range, in contrast to the values of $\tilde{R} b_{c r}$ which reduces sharply for the larger gyrotaxis ranges. Moreover, the growth in Péclet number is found to be sustaining the marginal changes in the wave numbers when these plots are compared (Figs. 3, 4). Overall the displayed critical wave numbers are smaller in magnitude for the oscillatory convection when compared to that of monotonic modes for the similar values of $R a$ [22]. At the cross-over points (Figs. 1, 2), bounces are reported in the corresponding wave numbers (Figs. 3,4 ) which clearly demarcates the bioconvection and traditional double diffusive convection where in the latter no jumps are there in the wave length or frequency $[21,41]$.

Figures 5 and 6 , illustrate the variation of vibrational parameter for the non-oscillatory and oscillatory

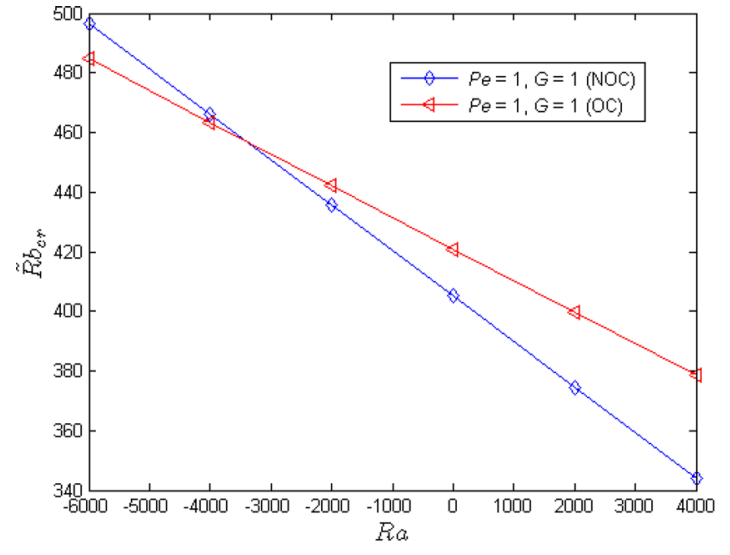

(a) modes on the $\left(R a, \tilde{R} b_{c r}\right)$ planes. In Fig. $5 \mathrm{a}, \mathrm{b}$ the results are depicted for the rigid-rigid boundary case while Fig. 6a, b, display the similar studies for a suspension with stress-free upper wall. Within the considered range of the other parameters the curves are traced out for the growing vibrational Rayleigh number $(R v=0,1000,5000)$. For both cases the bigger vibrational number exerts the higher magnitudes of $\tilde{R} b_{c r}$. This establishes the stabilizing nature of the high frequency vibration on the system [24]. Comparing the rigid-rigid and rigid-free cases (Figs. 5, 6), it is noticed that the strength of bioconvection remains almost unaltered irrespective of the modes of convection. Moreover, for increasing temperature gradient the slopes of the corresponding curves in the stationary modes are slightly quicker than the oscillatory modes of instability [21].

In Figs. 7 and 8 , the results on the $\left(R a, \tilde{R} b_{c r}\right)$ planes are displayed for the rigid-rigid boundary and the rigid-free surfaces, respectively. For the increasing $R v_{b}$, in the rigid-rigid case, the instability curves in the Fig. 7a,

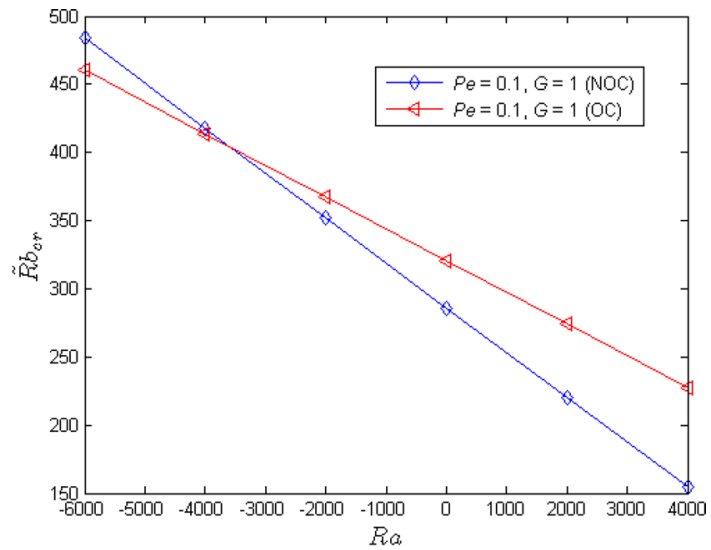

(b)

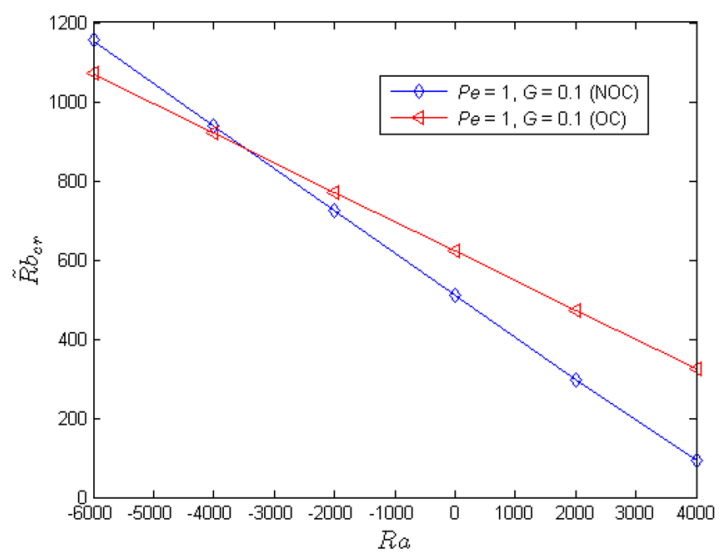

(c)

Fig. 2 The case of rigid lower and stress free upper surfaces: a-c Boundaries of Stationary and oscillatory instability modes of convections in the $\left(R a, \tilde{R} b_{c r}\right)$ plane for varying values of Péclet number Pe and Gyrotactic number $G$ 


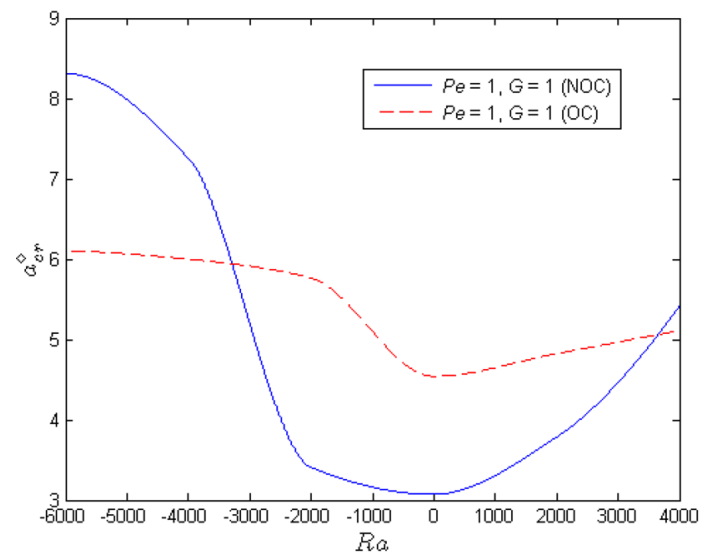

(a)

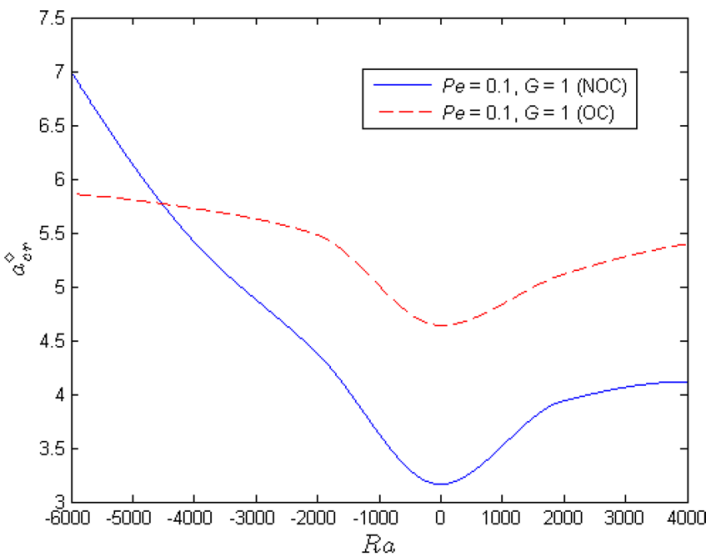

(b)

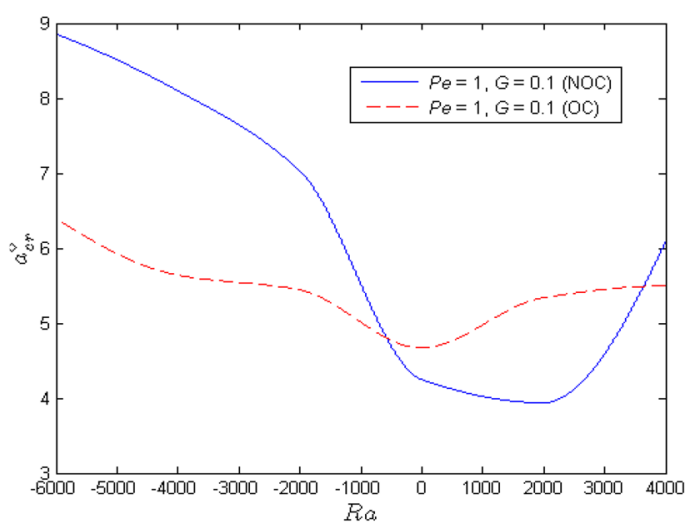

(c)

Fig. 3 The case of rigid upper and rigid lower walls: a-c Variation of thermal Rayleigh number verses critical wave number for the non-oscillatory and oscillatory modes of instability for the different values of Péclet number Pe and Gyrotactic number $G$

$b$ are referred to the stationary and oscillatory modes of convection, respectively. In Fig. $8 a$, b, the similar curves are depicted for the rigid-free boundaries. The parameter $R v_{b}$ defines the thermal and vibrational properties of the suspension jointly. For large negative $R a$, the observed $\tilde{R} b_{c r}$ suppresses with increase in $R v_{b}$ values. However, for the positive values of $R a$ the higher values of $R v_{b}$, corresponds to the higher magnitudes of $\tilde{R} b_{c r}$. Therefore, it is established that for the negative thermal gradient the suspension is dominated by the thermal properties of $R v_{b}$ while for the positive values of $R a$ its vibrational part becomes significantly dominant. Overall, varying the thermal effect $R v_{b}$ induces the destabilizing behavior to the system. On comparing the Figs. 7 and 8 ; it is reported that the values of $\tilde{R} b_{c r}$ are considerably deviated (irrespective of trends) for both the modes of convections. Particularly, for the positive thermal gradient $(R a>0)$ the slopes of the corresponding curves in stationary modes are found to be considerably quicker than the oscillatory modes of convection. This significance of the thermal and vibrational effects is supported by earlier published studies [21,24].

In Fig. 9, the dependence of the frequency of oscillation $\omega$ on $R a$ is displayed for the growing gyrotactic number (for fixed $\mathrm{Pe}=0.1$ ). Figure $9 \mathrm{a}$, b refers to the rigid-rigid and rigid-free modes of boundaries, respectively. In both of the cases, it is observed that for the increasing negative $R a$, the frequency $\omega$ gets bigger from a finite value. On comparing Fig. 9 a, $b$ the magnitudes of frequency $\omega$ slightly strengthens with the increase in gyrotaxis effect, for the similar thermal variation across the suspension. Therefore, for the slower swimmers the frequency of oscillation 


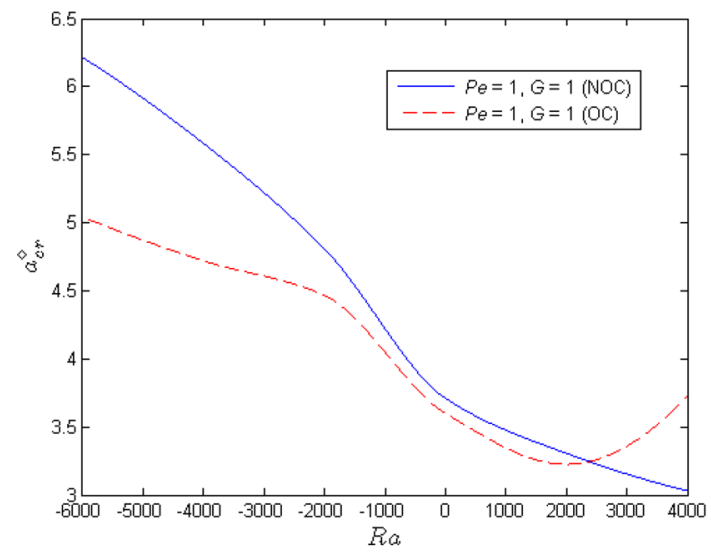

(a)

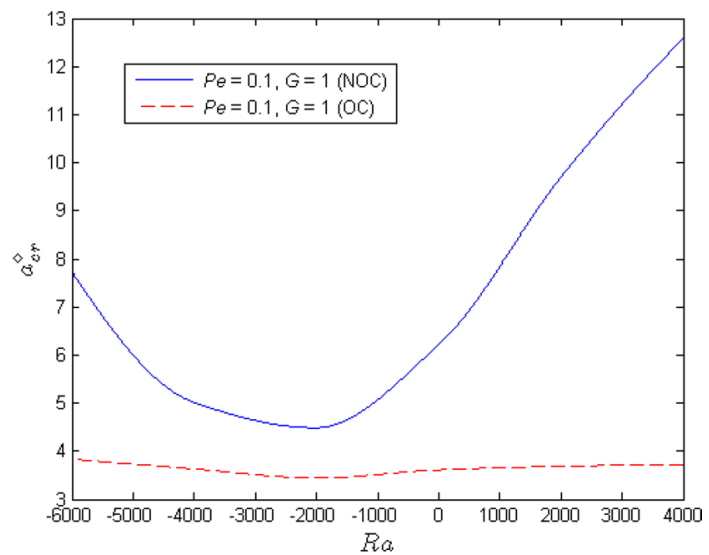

(b)

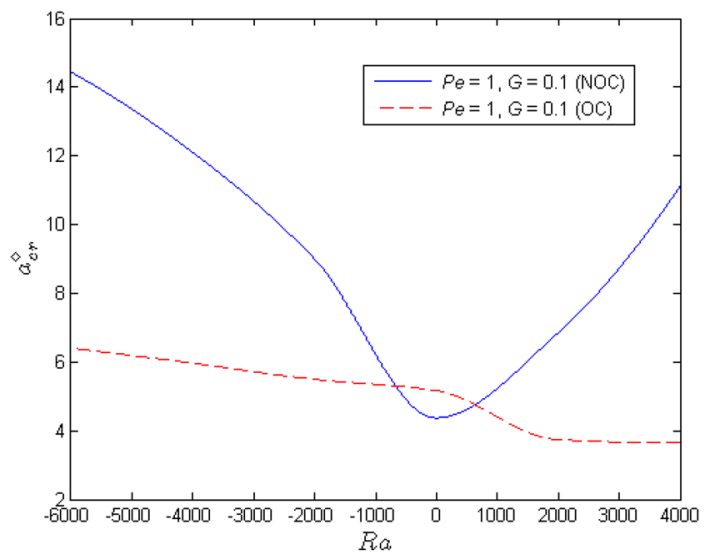

(c)

Fig. 4 The case of rigid lower and stress free upper walls: a-c Variation in thermal Rayleigh number verses critical wave number for the nonoscillatory and oscillatory modes of instability for the different values of gyrotactic number $G$ and Péclet number $P e$

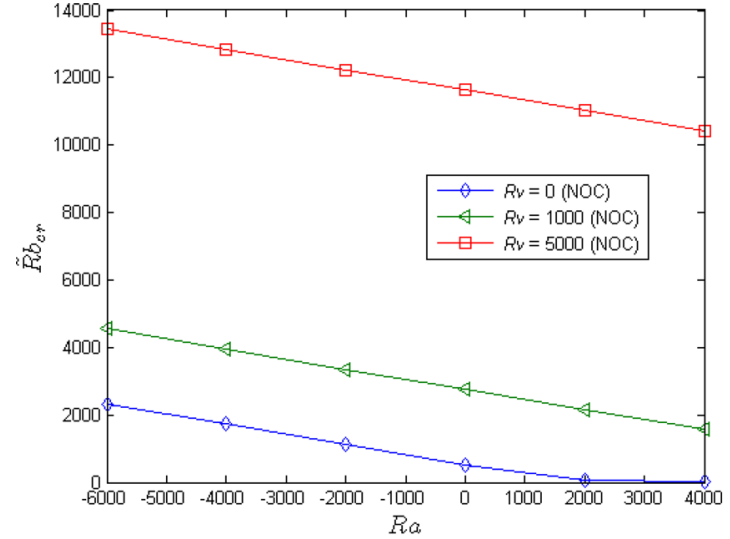

(a)

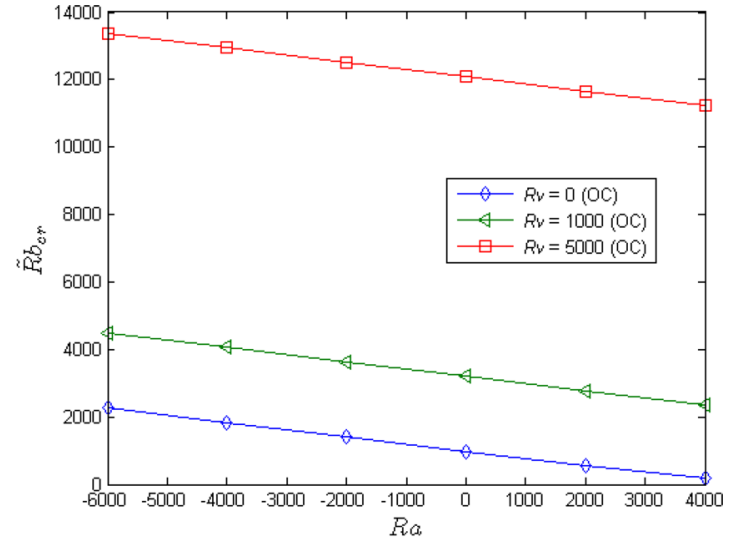

(b)

Fig. 5 The case of rigid upper and rigid lower surfaces: $\mathbf{a}$, b Boundaries of stationary and oscillatory instabilities in the $\left(R a, \tilde{R} b_{c r}\right)$ plane for the varying values of vibrational Rayleigh number $R v$ 


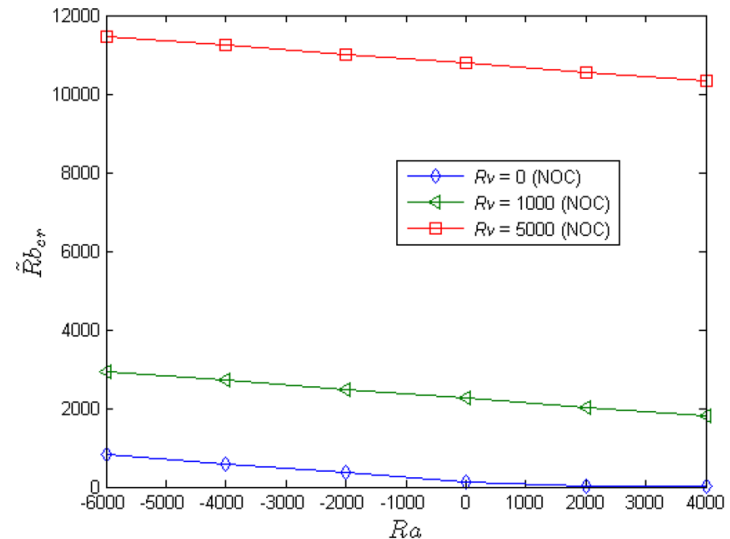

(a)

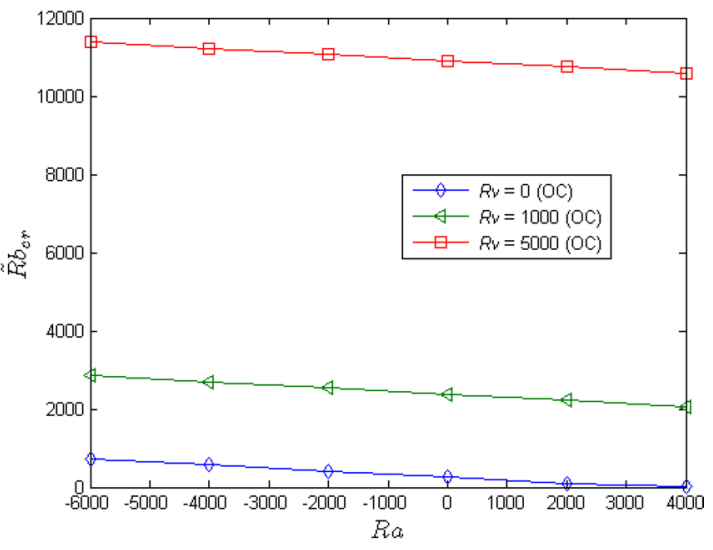

(b)

Fig. 6 The case of rigid lower and stress free upper surfaces: $\mathbf{a}, \mathbf{b}$ Boundaries of stationary and oscillatory convections in the $\left(R a, \tilde{R} b_{c r}\right)$ plane for the varying values of vibrational Rayleigh number $R v$

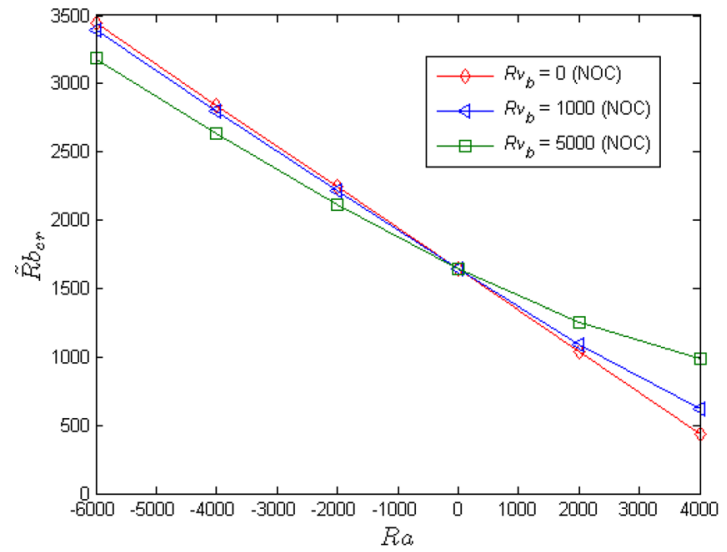

(a)

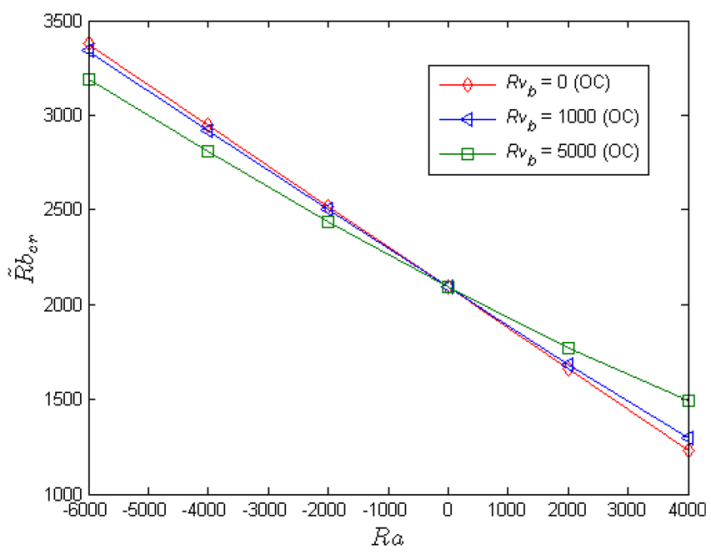

(b)

Fig. 7 The case of rigid lower and rigid upper surfaces: $\mathbf{a}, \mathbf{b}$ Boundaries of stationary and oscillatory modes of convections in the $\left(R a, \tilde{R} b_{c r}\right)$ plane for the various values of thermo-vibrational parameter $R v_{b}$

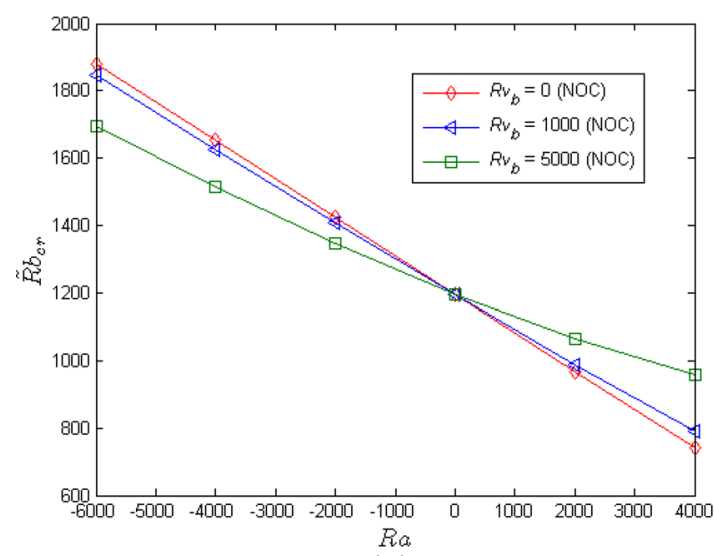

(a)

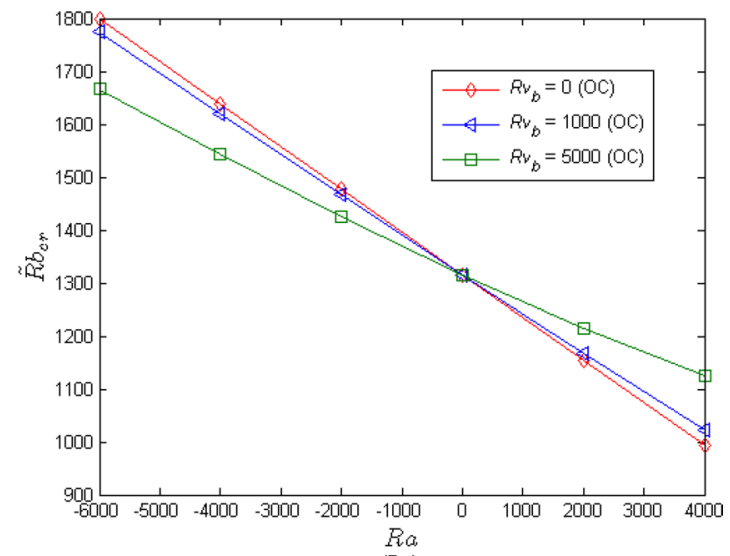

(b)

Fig. 8 The case of rigid lower and stress free upper surfaces: $\mathbf{a}, \mathbf{b}$ Boundaries of stationary and oscillatory modes of convections in the $\left(R a, \tilde{R} b_{c r}\right)$ plane for the various values of thermo-vibrational parameter $R v_{b}$ 


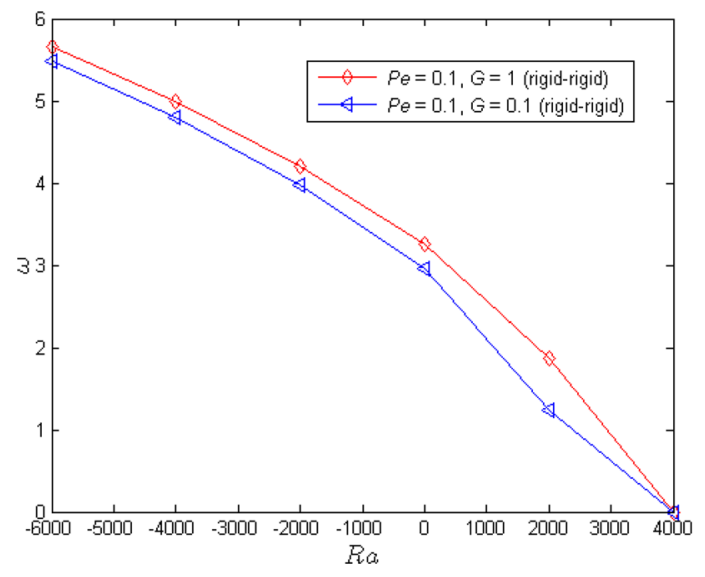

(a)

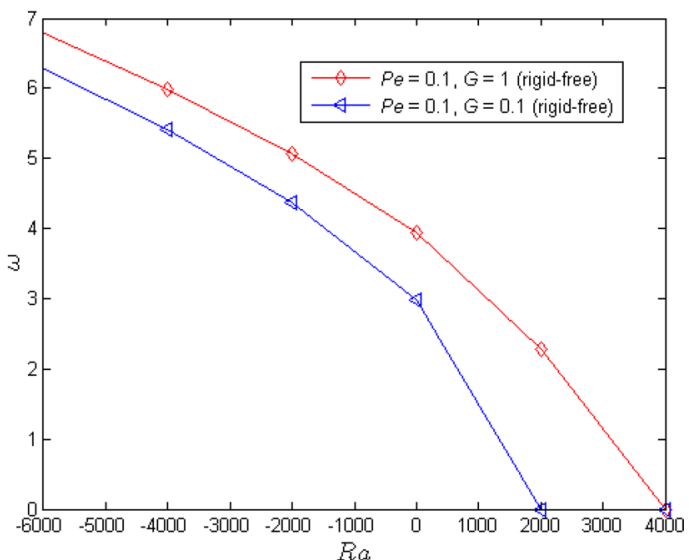

(b)

Fig. 9 The case of rigid-rigid and rigid-free walls: $\mathbf{a}, \mathbf{b}$ Boundaries of oscillatory mode of instability in the $(R a, \omega)$ plane for the various values of gyrotactic number $G$ and Péclet number $P e$

corresponds to the marginal deviation subjected to the variation in gyrotactic number [21].

\section{Funding sources}

This research did not receive any specific grant from funding agencies in the public, commercial, or not-for-profit sectors.

\section{Conclusions}

We have performed an overstability analysis of a thermo-vibrational convection with a clear physical and mathematical context to describe the heat transfer phenomena in a gyrotactic suspension. The results display the influence of vertical vibration and thermal gradient on the gyrotactic patterns analytically. For a negative thermal gradient with a nonzero Péclet number overstability occurs as a special mode of convection. Thermal variation across the surfaces prompts the reduction in the bioconvection and thus the system is destabilized in both of oscillatory and non-oscillatory modes of convection. The reported $\tilde{R} b_{c r}$ is superior for higher values of Péclet number whose stabilizing effect is pronounced. Gyrotaxis prompts the diminution in the critical bioconvection Rayleigh number and consequently destabilizes the system. The bioconvection strength is found higher for the bigger vibrational Rayleigh number and thus stabilization is provoked by induced vibration. The destabilizing impact of the thermo-vibrational parameter is due to its thermal properties for a suspension cooling from below. Moreover, an adequate stabilizing nature is also depicted due to its vibrational component when the thermal stratification is positive. It is summarized that the suspension with rigid-rigid boundaries is more stable than the one with stress-free top layer. Future development of this research should address a more rigorous weakly nonlinear convection.

\section{Declarations}

Conflict of interest No potential conflict of interest was reported by authors.

Open Access This article is licensed under a Creative Commons Attribution 4.0 International License, which permits use, sharing, adaptation, distribution and reproduction in any medium or format, as long as you give appropriate credit to the original author(s) and the source, provide a link to the Creative Commons licence, and indicate if changes were made. The images or other third party material in this article are included in the article's Creative Commons licence, unless indicated otherwise in a credit line to the material. If material is not included in the article's Creative Commons licence and your intended use is not permitted by statutory regulation or exceeds the permitted use, you will need to obtain permission directly from the copyright holder. To view a copy of this licence, visit http://creativecommons. org/licenses/by/4.0/.

\section{Appendices}

\section{Appendix 1}

The functions $\chi_{1} \chi_{1}, \chi_{2}$ and $\chi_{3}$ defined in Eq. (29) are

$\chi=\left(1+a^{\diamond^{2}}\left(1-\alpha_{0}\right) G\right) \chi_{1}-\left(1+\alpha_{0}\right) G \chi_{2}$ 
$\chi_{1}=\left(52 /(P e)^{3}+528 /(P e)^{5}\right)(\exp (P e)-1)-\left(4 /(P e)^{2}+264 /(P e)^{4}\right)(\exp (P e)+1)$

$\chi_{2}=\left(16 /(P e)+192 /(P e)^{3}\right)(\exp (P e)-1)-\left(96 /(P e)^{2}\right)(\exp (P e)+1)$

$\chi_{3}=\left(72 /(P e)^{5}+720 /(P e)^{7}\right)(\exp (P e)-1)-\left(6 /(P e)^{4}+360 /(P e)^{6}\right)(\exp (P e)+1)$

The symbols $\alpha_{i}, \beta_{i}(i=1,2, \ldots 7)$ defined in Eq. (30) are as follows

$\alpha_{1}=1260 a^{{\vartheta^{2}}^{2}} \chi\left(28-3(P e)^{2}\right)\left(10+a^{{\vartheta^{2}}^{2}}\right)^{2}, \quad \beta_{1}=1260 a^{{\diamond^{2}}^{2}} \chi\left(28-3(P e)^{2}\right)\left(10+a^{{\vartheta^{2}}^{2}}\right) \operatorname{Pr}$

$\left.\begin{array}{l}\alpha_{2}=27 a^{\diamond^{2}}\left[a^{\diamond^{2}}\left(120-10(P e)^{2}+(P e)^{4}\right)+10(P e)^{4}\right]\left(10+a^{\diamond^{2}}\right) \\ \beta_{2}=27 a^{\diamond^{2}} L e \operatorname{Pr}\left[\left(120-10(P e)^{2}+(P e)^{4}\right)\right]\left(10+a^{\diamond^{2}}\right)\end{array}\right\}$

$\alpha_{3}=529200 a^{\diamond^{4}} \chi \chi_{3}\left(10-(P e)^{2}\right)\left(10+a^{\diamond^{2}}\right), \quad \beta_{3}=529200 a^{\diamond^{4}} \chi \chi_{3}\left(10-(P e)^{2}\right) \operatorname{Pr}$

$\alpha_{4}=27 a^{\diamond^{4}}\left[a^{\diamond^{2}}\left(120-10(P e)^{2}+(P e)^{4}\right)+10(P e)^{4}\right], \quad \beta_{4}=27 a^{\diamond^{2}} L e \operatorname{Pr}\left[\left(120-10(P e)^{2}+(P e)^{4}\right)\right]$

$\alpha_{5}=28\left(10+a^{\diamond^{2}}\right)\left(a^{\diamond^{4}}+24 a^{\diamond^{2}}+504\right), \quad \beta_{5}=28\left(10+a^{\diamond^{2}}\right)\left(12+a^{\diamond^{2}}\right)$

$\alpha_{6}=a^{\diamond^{2}}\left(120-10(P e)^{2}+(P e)^{4}\right)+10(P e)^{4}, \beta_{6}=L e \operatorname{Pr}\left(120-10(P e)^{2}+(P e)^{4}\right), \alpha_{7}=\left(10+a^{\diamond^{2}}\right), \beta_{7}=\operatorname{Pr}$

The closed forms of the expressions $R b_{0}, R a_{0}, R v_{0}$ and $R a_{v b_{0}}$ defined in Eq. (32) as $P e \rightarrow 0$ and $G \rightarrow 0$ are:

$R b_{0}=10\left(a^{\diamond^{4}}+24 a^{\diamond^{2}}+504\right) / 7, \quad R a_{0}=28\left(10+a^{\diamond^{2}}\right)\left(a^{\diamond^{4}}+24 a^{\diamond^{2}}+504\right) / 27 a^{\diamond^{2}}$

$R v_{0}=1764\left(10+a^{\diamond^{2}}\right)\left(a^{\diamond^{4}}+24 a^{\diamond^{2}}+504\right) / 1323 a^{\diamond^{2}}, \quad R a_{v b_{0}}=28\left(10+a^{{\vartheta^{2}}^{2}}\right)^{2}\left(a^{\diamond^{4}}+24 a^{\diamond^{2}}+504\right) / 27 a^{\diamond^{4}}$

For the limiting when $P e \rightarrow 0$ and $G \rightarrow 0$, the values of $\Lambda_{i}(i=1,2, \ldots 7)$ defined in Eq. (38) are given as:

$\Lambda_{1}=\Lambda_{3}=\Lambda_{7}=\operatorname{Pr} /\left(10+a^{\diamond^{2}}\right), \quad \Lambda_{2}=\Lambda_{4}=\Lambda_{6}=L e \operatorname{Pr} / a^{\diamond^{2}}, \quad \Lambda_{7}=\left(12+a^{\diamond^{2}}\right) /\left(a^{\diamond^{4}}+24 a^{\diamond^{2}}+504\right)$ 


\section{Appendix 2}

$$
\left.\begin{array}{l}
R v_{1}=40\left(19 a^{\diamond^{4}}+432 a^{\diamond^{2}}+4536\right)\left(10+a^{\diamond^{2}}\right) / 567 a^{\diamond^{2}} \\
R a_{v b_{1}}=28\left(19 a^{\diamond^{4}}+432 a^{\diamond^{2}}+4536\right)\left(10+a^{\diamond^{2}}\right)^{2} / 507 a^{\diamond^{4}}
\end{array}\right\}
$$

$\zeta=\left(1+a^{\diamond^{2}}\left(1-\alpha_{0}\right) G\right) \zeta_{1}-\left(1+\alpha_{0}\right) G \zeta_{2}$

For the limiting when $P e \rightarrow 0$ and $G \rightarrow 0$, the values of $\Lambda_{1 i}(i=1,2, \ldots 7)$ defined in Eq. (50) are given as

$\zeta_{1}=\left(1056 /(P e)^{5}\right)(\exp (P e)-1)-\{444 \exp (P e)+612\} /(P e)^{4}+\{54 \exp (P e)-138\} /(P e)^{3}+\{4 \exp (P e)-12\} /(P e)^{2}-\exp (P e) /(P e)$

$\zeta_{2}=6\left[\{64(\exp (P e)-1)\} /(P e)^{3}-\{28 \exp (P e)+36\} /(P e)^{2}+\{3 \exp (P e)-7\} /(P e)\right]$

$\zeta_{3}=\{1440(\exp (P e)-1)\} /(P e)^{7}-\{600 \exp (P e)+840\} /(P e)^{6}+\{72 \exp (P e)-192\} /(P e)^{5}+\{6 \exp (P e)-18\} /(P e)^{4}-2 \exp (P e) /(P e)^{3}$

The functions $\alpha_{1 i}, \beta_{1 i}(i=1,2, \ldots 7)$ defined in Eq. (43) are:

$\alpha_{11}=1260 a^{\diamond^{2}} \zeta\left[126+7 P e-13(P e)^{2}\right]\left(10+a^{\diamond^{2}}\right)^{2}, \quad \beta_{11}=1260 a^{\diamond^{2}} \zeta\left[126+7 P e-13(P e)^{2}\right]\left(10+a^{\diamond^{2}}\right) \operatorname{Pr}$

$\left.\begin{array}{l}\alpha_{12}=507 a^{\diamond^{2}}\left[a^{\diamond^{2}}\left(120-10(P e)^{2}+(P e)^{4}\right)+10(P e)^{4}\right]\left(10+a^{\diamond^{2}}\right) \\ \beta_{12}=507 a^{\diamond^{2}}\left[\left(120-10(P e)^{2}+(P e)^{4}\right)\right]\left(10+a^{\diamond^{2}}\right) L e \operatorname{Pr}\end{array}\right\}$

$$
\left.\begin{array}{l}
\Lambda_{11}=\Lambda_{13}=\Lambda_{15}=\operatorname{Pr} /\left(10+a^{\diamond^{2}}\right), \quad \Lambda_{12}=\Lambda_{14}=\Lambda_{17}=\text { LePr } / a^{\diamond^{2}}, \\
\Lambda_{16}=\left(19 a^{\diamond^{2}}+216\right) /\left(19 a^{\diamond^{4}}+432 a^{\diamond^{2}}+4536\right)
\end{array}\right\}
$$

$\alpha_{13}=529200 a^{\diamond^{4}} \zeta \zeta_{3}\left(10-(P e)^{2}\right)\left(10+a^{\diamond^{2}}\right), \quad \beta_{13}=529200 a^{\diamond^{4}} \zeta \zeta_{3}\left(10-(P e)^{2}\right) \operatorname{Pr}$

$\alpha_{14}=507 a^{\diamond^{4}}\left[a^{\diamond^{2}}\left(120-10(P e)^{2}+(P e)^{4}\right)+10(P e)^{4}\right], \quad \beta_{14}=507 a^{\diamond^{4}}\left[\left(120-10(P e)^{2}+(P e)^{4}\right)\right] L e P r$

$\alpha_{15}=28\left(10+a^{\diamond^{2}}\right)^{2}, \quad \beta_{15}=28\left(10+a^{\diamond^{2}}\right) \operatorname{Pr}$

$\left.\begin{array}{l}\alpha_{16}=19 a^{\diamond^{4}}+432 a^{\diamond^{2}}+4536, \quad \alpha_{17}=\left[a^{\diamond^{2}}\left(120-10(P e)^{2}+(P e)^{4}\right)+10(P e)^{4}\right] \\ \beta_{16}=19 a^{\diamond^{2}}+216, \quad \beta_{17}=\left(120-10(P e)^{2}+(P e)^{4}\right) L e P r\end{array}\right\}$

The closed form of the expressions $R b_{1}, R a_{1}, R v_{1}$ and $R a_{v b_{1}}$ defined in Eq. (45) as $P e \rightarrow 0$ and $G \rightarrow 0$ are given: $\quad R b_{1}=40\left(19 a^{\diamond^{4}}+432 a^{\diamond^{2}}+4536\right) / 567, \quad R a_{1}$ $=28\left(10+a^{\diamond^{2}}\right)\left(19 a^{\diamond^{4}}+432 a^{\diamond^{2}}+4536\right) / 507 a^{\diamond^{2}}(74)$ 


\section{References}

1. Childress S, Levandowsky M, Spiegel EA (1975) Pattern formation in a suspension of swimming micro-organisms: equations and stability theory. J Fluid Mech 69:591-613

2. Pedley TJ, Hill NA, Kessler JO (1988) The growth of bioconvection patterns in a uniform suspension of gyrotactic microorganisms. J Fluid Mech 195:223-237

3. Bees MA, Hill NA (1998) Linear bioconvection in a suspension of randomly swimming, gyrotactic micro-organisms. Phys Fluids 10(8):1864-1881

4. Ishikawa T, Pedley TJ (2007) Diffusion of swimming model micro-organisms in a semi-dilute suspension. J Fluid Mech 588:437-462

5. Sen $\mathrm{R}$ (2008) Biotechnology in petroleum recovery: The microbial EOR. Prog Energy Combust Sci 34:714-724

6. Gao CH, Zekri A (2011) Applications of microbial-enhanced oil recovery technology in the past decade. Energy Sources Part A Recovery Utili Environ Eff 33:972-989

7. Ghorai S, Singh R (2009) Linear stability analysis of gyrotactic plumes. Phys Fluids 21:081901

8. Pedley TJ (2010) Instability of uniform microorganism suspensions revisited. J Fluid Mech 647:335-359

9. Hwang Y, Pedley TJ (2014) Bioconvection under uniform shear: linear stability analysis. J Fluid Mech 738:522-562

10. Hwang Y, Pedley TJ (2014) Stability of down flowing gyrotactic microorganism suspensions in a two-dimensional vertical channel. J Fluid Mech 749:750-777

11. Kuznetsov AV (2005) The onset of bioconvection in a suspension of gyrotactic microorganisms in a fluid layer of finite depth heated from below. Int Commun Heat Mass Transfer 32:574-582

12. Gudiña EJ, Pereira JFB, Costa R, Coutinho JAP, Teixeira JA, Rodrigues LR (2013) Biosurfactant-producing and oil-degrading bacillus subtilis strains enhance oil recovery in laboratory sandpack columns. J Hazard Mater 261:106-113

13. Hong E, Jeong MS, Kim TH, Lee JH, Cho JH, Lee KS (2019) Development of coupled biokinetic and thermal model to optimize cold-water microbial enhanced oil recovery (meor) in homogenous reservoir. Sustainability 11:1652

14. Simonenko IB, Zen'kovskaya SM, (1966) Effect of high frequency vibration on convection initiation. Izv Akad Nauk SSSR, Mekh Zhidk Gaza 1:51-55

15. Wolf GH (1970) Dynamic stabilization of the interchange instability of a liquid-gas interface. Phys Rev Lett 24:444

16. Lyubimov DV (1995) Convective flows under the influence of high-frequency vibrations. Eur J Mech B Fluids 14(4):439-458

17. Gershuni GZ, Lyubimov DU (1998) Thermal vibrational convection. Wiley, New York

18. Zen'kovskaya SM, Novosyadlyi VA, (2009) The effect of a highfrequency progressive vibration on the convective instability of a two-layer fluid. J Appl Math Mech 73:271-280

19. Boffetta G, Magnani M, Musacchio S (2019) Suppression of Rayleigh-Taylor turbulence by time-periodic acceleration. Phys Rev E 99:033110

20. Kuznetsov AV (2005) The onset of bioconvection in a suspension of negatively geotactic microorganisms with high-frequency vertical vibration. Int Commun Heat Mass Transfer 32:1119-1127

21. Nield DA, Kuznetsov AV (2006) The onset of bio-thermal convection in a suspension of gyrotactic microorganisms in a fluid layer: oscillatory convection. Int J Therm Sci 45:990-997

22. Sharma YD, Kumar V (2011) Overstability analysis of thermobioconvection saturating a porous medium in a suspension of gyrotactic microorganisms. Transp Porous Media 90:673-685
23. Kuznetsov AV (2011) Non-oscillatory and oscillatory nanofluid bio-thermal convection in a horizontal layer of finite depth. Eur J Mech B Fluids 30:156-165

24. Sharma YD, Kumar V (2012) The effect of high-frequency vertical vibration in a suspension of gyrotactic micro-organisms. Mech Res Commun 44:40-46

25. Kumar V, Sharma YD (2014) Instability analysis of gyrotactic microorganisms: a combined effect of high-frequency vertical vibration and porous media. Transp Porous Media 102:153-165

26. Becker AD, Masoud H, Newbolt JW, M, Shelley, L, Ristroph (2015) Hydrodynamic schooling of flapping swimmers. Nat Commun 6:8514

27. Bechinger $C$, Di Leonardo R, Löwen $H$, Reichhardt $C$, Volpe $G$, Volpe $G$ (2016) Active particles in complex and crowded environments. Rev Mod Phys 88:045006

28. Uddin MJ, Alginahi Y, Beg OA, Kabir MN (2016) Numerical solutions for nonlinear gyrotactic bioconvection in nanofluid saturated porous media with stefan blowing and multiple slip effects. Comput Math Appl 10:2562-2581

29. Lovecchio S, Zonta F, Marchioli C, Soldati A (2017) Thermal stratification hinders gyrotactic micro-organism rising in free-surface turbulence. Phys Fluids 29:053302

30. Xun S, Zhao J, Zheng L, Zhang X (2017) Bioconvection in rotating system immersed in nanofluid with temperature dependent viscosity and thermal conductivity. Int J Heat Mass Transfer 111:1001-1006

31. Zhao M, Xiao Y, Wang S (2018) Linear stability of thermal-bioconvection in a suspension of gyrotactic micro-organisms. Int J Heat Mass Transfer 126:95-102

32. Mashayekhpour M, Marchioli C, Lovecchio S, Nemati Lay E, Soldati A (2019) Wind effect on gyrotactic micro-organism surfacing in free-surface turbulence. Adv Water Resour 129:328-337

33. Marchioli C, Bhatia H, Sardina G, Brandt L, Soldati A (2019) Role of large-scale advection and small-scale turbulence on vertical migration of gyrotactic swimmers. Phys Rev Fluids 4:124304

34. Nayak MK, Prakash J, Tripathi D, Pandey VS, Shaw S, Makinde OD (2020) 3D Bioconvective multiple slip flow of chemically reactive Casson nanofluid with gyrotactic micro-organisms. Heat Transfer-Asian Research 49:135-153

35. Bees MA (2020) Advances in bioconvection. Annu Rev Fluid Mech 52:449-476

36. Bardan G, Razi YP, Mojtabi A (2004) Comments on the mean flow averaged model. Phys Fluids 16:4535-4538

37. Razi YP, Charrier-Mojtabi MC, Mojtabi A (2008) Thermal vibrational convection in a porous media saturated by a pure or binary fluid. In: Vadasz P (ed) Emerging topics in heat and mass transfer in porous media. Springer, Dordrecht, pp 149-179

38. Chandrasekhar S (1981) Hydrodynamic and hydromagnetic stability. Dover, New York

39. Finlayson BA (1972) The method of weighted residuals and variational principles. Academic Press, New York

40. Sparrow EM, Goldstein RJ, Jonsson VK (1964) Thermal instability in a horizontal fluid layer: effect of boundary conditions and non-linear temperature profile. J Fluid Mech 18:513-528

41. Nield DA (1967) The thermohaline Rayleigh-Jeffreys problem. J Fluid Mech 29:545-558

Publisher's Note Springer Nature remains neutral with regard to jurisdictional claims in published maps and institutional affiliations. 